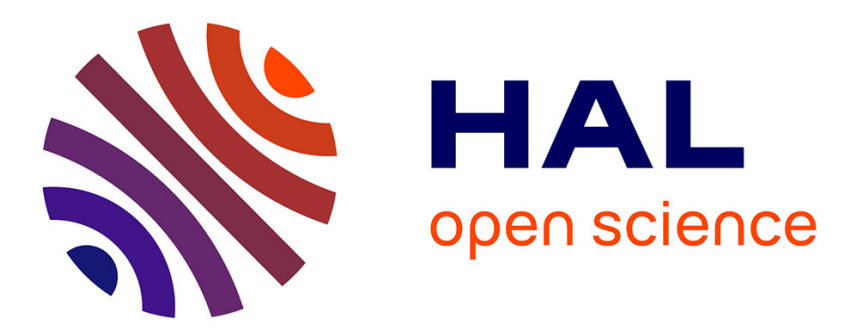

\title{
Ab initio potential energy curve for the neon atom pair and thermophysical properties for the dilute neon gas. II. Thermophysical properties for low-density neon
} Eckhard Vogel, Eckard Bich, Robert Hellmann

\section{- To cite this version:}

Eckhard Vogel, Eckard Bich, Robert Hellmann. Ab initio potential energy curve for the neon atom pair and thermophysical properties for the dilute neon gas. II. Thermophysical properties for low-density neon. Molecular Physics, 2008, 106 (06), pp.813-825. 10.1080/00268970801964207 . hal-00513181

\author{
HAL Id: hal-00513181 \\ https://hal.science/hal-00513181
}

Submitted on 1 Sep 2010

HAL is a multi-disciplinary open access archive for the deposit and dissemination of scientific research documents, whether they are published or not. The documents may come from teaching and research institutions in France or abroad, or from public or private research centers.
L'archive ouverte pluridisciplinaire HAL, est destinée au dépôt et à la diffusion de documents scientifiques de niveau recherche, publiés ou non, émanant des établissements d'enseignement et de recherche français ou étrangers, des laboratoires publics ou privés. 


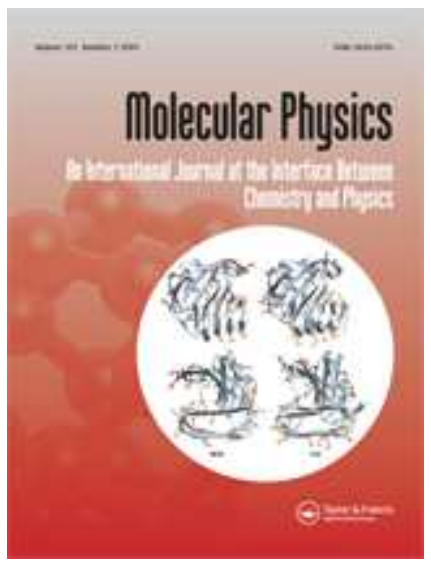

\section{Ab initio potential energy curve for the neon atom pair and thermophysical properties for the dilute neon gas. II. Thermophysical properties for low-density neon}

\begin{tabular}{|r|l|}
\hline Journal: & Molecular Physics \\
\hline Manuscript ID: & TMPH-2007-0366.R1 \\
\hline Manuscript Type: & Full Paper \\
\hline Author: & 04-Feb-2008 \\
\hline Complete List of Authors: & $\begin{array}{l}\text { Vogel, Eckhard; Universität Rostock, Institut für Chemie; } \\
\text { Universität Rostock, Institut für Chemie } \\
\text { Bich, Eckard; Universität Rostock, Institut für Chemie } \\
\text { Hellmann, Robert; Universität Rostock, Institut für Chemie }\end{array}$ \\
\hline Keywords: & $\begin{array}{l}\text { Neon pair potential, neon gas property standards, second and third } \\
\text { pressure virial coefficients, viscosity, thermal conductivity }\end{array}$ \\
\hline $\begin{array}{l}\text { Note: The following files were submitted by the author for peer review, but cannot be converted } \\
\text { to PDF. You must view these files (e.g. movies) online. }\end{array}$ \\
\hline Neon2rev1-MolPhys.tex \\
\hline \hline
\end{tabular}

\section{S ScholaroNE \\ Manuscript Central}




\title{
$A b$ initio potential energy curve for the neon atom pair and thermophysical properties for the dilute neon gas. \\ II. Thermophysical properties for low-density neon
}

\author{
ECKARD BICH, ROBERT HELLMANN, and ECKHARD VOGEL* \\ Institut für Chemie, Universität Rostock, Albert-Einstein-Straße 3a, D-18059 Rostock, \\ Germany \\ (Received 00 Month 200x; in final form 00 Month 200x)
}

\begin{abstract}
A neon-neon interatomic potential energy curve determined from quantum-mechanical $a b$ initio calculations and described with an analytical representation (R. Hellmann, E. Bich, and E. Vogel, Molec. Phys. (in press)) was used in the framework of the quantum-statistical mechanics and of the corresponding kinetic theory to calculate the most important thermophysical properties of neon governed by two-body and three-body interactions. The second and third pressure virial coefficients as well as the viscosity and thermal conductivity coefficients, the last two in the so-called limit of zero density, were calculated for natural Ne from $25 \mathrm{~K}$ to $10,000 \mathrm{~K}$. Comparison of the calculated viscosity and thermal conductivity with the most accurate experimental data at ambient temperature shows that these values are accurate enough to be applied as standard values for the complete temperature range of the calculations characterized by an uncertainty of about $\pm 0.1 \%$ except at the lowest temperatures.
\end{abstract}

Keywords: Neon pair potential; neon gas property standards; second and third pressure virial coefficients; viscosity; thermal conductivity.

\section{Introduction}

Recently we calculated standard values for some thermophysical two-body and three-body properties of helium over the temperature range from $1 \mathrm{~K}$ to $10,000 \mathrm{~K}$ with uncertainties that are superior to experimental data [1]. Prerequisite for it was the determination of a state-of-the-art pair potential between two helium atoms [2]. In order to establish a second standard for the calibration of high-precision measuring instruments at low density, we developed very recently a new interatomic pair potential for neon from high-level supermolecular ab initio calculations for a large number of internuclear separations $R$ (paper I [3]). The ab initio calculated interatomic potential energy values $V(R)$, including core-core and core-valence correlation and relativistic corrections as well as coupled-cluster contributions up to

*Corresponding author. Email: eckhard.vogel@uni-rostock.de 
CCSDT(Q), were listed in Table 2 of paper I. They were used for the fit of an analytical potential function, which represents a modification of the potential function given by Tang and Toennies [4]:

$$
\begin{aligned}
V(R) & =A \exp \left(a_{1} R+a_{2} R^{2}+a_{-1} R^{-1}+a_{-2} R^{-2}\right) \\
& -\sum_{n=3}^{8} \frac{C_{2 n}}{R^{2 n}}\left[1-\exp (-b R) \sum_{k=0}^{2 n} \frac{(b R)^{k}}{k !}\right] .
\end{aligned}
$$

Whereas the details of the fit were communicated in paper I, the potential parameters are repeated here for convenience in Table 1.

A comparison in paper I with experimental rovibrational spectra [5] showed that the new potential function is superior to the $a b$ initio potential by Cybulski and Toczylowski [6]. This potential was given as an analytical function derived from $a b$ initio values calculated for a large range of internuclear distances. Furthermore, the comparison made evident that our new potential is at least as good as the best semi-empirical potential by Aziz and Slaman [7] and also compares well with the potential of Wüest and Merkt [5] fitted directly to the rovibrational spectra under discussion. It is noteworthy that the rovibrational spectra are sensitive to the shape of the potential well. Hence it could be possible that the potential of Wüest and Merkt is not so effective with respect to other regions of the potential. On the contrary, the transport properties are particularly sensitive to the repulsive part of the potential. Thus the potential of Aziz and Slaman could be expected to perform well in nearly all regions of the potential, since it was determined in a fit to high-energy beam data and to viscosity coefficients, considering calculated values of the $C_{6}, C_{8}$ and $C_{10}$ dispersion coefficients.

In this paper we report standard values of the second and third pressure virial coefficients as well as of the viscosity and thermal conductivity coefficients in the limit of zero density for neon in its natural isotopic composition. Even though the quality of the new neon ab initio potential of paper I [3] is somewhat inferior compared with our recent $a b$ initio potential for helium [2], the calculated thermophysical properties are expected to be as accurate as the best experimental measurements at room temperature and more accurate than available experimental data far above and far below room temperature. In order to assess as accurately as possible the quality of the potentials considered in this paper, some of the experimental viscosity data from the literature were recalibrated with reference values derived from the new interatomic potential for helium $[1,2]$. 


\section{Molecul}

\section{Quantum-mechanical calculation of thermophysical properties}

\subsection{Evaluation of the phase shifts}

A quantum-mechanical treatment of the elastic scattering is needed to obtain very accurate values for the thermophysical properties of neon. For this purpose the relative phase shifts $\delta_{l}(k)$ have to be evaluated as asymptotic limiting values of the relative phases of the perturbed and unperturbed radial factor wave functions $\psi_{l}(R)$ and $\psi_{l}^{(0)}(R)$ (the latter with $V(R)=0$ ). Each of them corresponds to a particular state of the angular momentum of the system characterized by the quantum number $l$. To obtain the relative phase shifts $\delta_{l}(k)$, the Schrödinger equation is to be solved by numerical integration for many values of the wave number $k=(2 \mu E)^{1 / 2} / \hbar$, where $E$ is the energy of the incoming wave and $\mu=\left(m_{1} m_{2}\right) /\left(m_{1}+m_{2}\right)$ is the reduced mass.

In principle, neon is to consider as a mixture of the three isotopes ${ }^{20} \mathrm{Ne},{ }^{21} \mathrm{Ne}$, and ${ }^{22} \mathrm{Ne}$ with the relative atomic masses 19.9924356, 20.9938428, and 21.9913831, with the nuclear spins $s$ of $0,3 / 2$, and 0 , and with the natural abundances 90.48 atom\%, 0.27 atom\%, and 9.27 atom\%. Hence there are six different interacting systems in naturally occurring neon with varying reduced masses and different statistics: ${ }^{20} \mathrm{Ne}-{ }^{20} \mathrm{Ne}$ and ${ }^{22} \mathrm{Ne}-{ }^{22} \mathrm{Ne}$ (both Bose-Einstein statistics), ${ }^{21} \mathrm{Ne}^{21} \mathrm{Ne}$ (Fermi-Dirac statistics), ${ }^{20} \mathrm{Ne}-{ }^{21} \mathrm{Ne},{ }^{20} \mathrm{Ne}-{ }^{22} \mathrm{Ne}$, and ${ }^{21} \mathrm{Ne}-{ }^{22} \mathrm{Ne}$ (all Boltzmann statistics). As a consequence, the relative phase shifts have to be calculated for these six binary systems at a multiplicity of wave numbers $k$ or of energies $E$ for a substantial number of $l$ values which requires plenty of computing time. In order to save time the semi-classical JWKB method was used as approximation. Problems and the procedure of the fully quantum-mechanical calculation as well as the JWKB method for the determination of the relative phase shifts were discussed in some detail in our paper on helium [1].

To avoid uncertainties in the results of the calculated thermophysical properties, a very large number of phase shifts $\delta_{l}(k)$ was determined for 500 values of the energy $E$ from zero to about $135,000 \mathrm{~K}$ and for an increasing number of $l$ values related to the energy. The calculations of the phase shifts were first performed fully quantum-mechanically and for comparison parallel to it according to the JWKB approximation. If the values resulting from both procedures became practically identical for certain values of the angular momentum quantum number $l$, the fully quantum-mechanical evaluation (QM) was finished and substituted by the semiclassical JWKB procedure at the higher $l$ values. Table 2 gives an overview about the number of phase shifts determined for some reduced energies $E^{*}=E / \varepsilon$. 


\subsection{Calculation of the second and third pressure virial coefficients}

In this paper two alternative ways were used to calculate the second virial coefficient of naturally occurring neon as a function of temperature $T$. In the first variant $B(T)$ is determined like that of a mixture composed of the three neon isotopes:

$$
B_{\text {mix }}(T)=\sum_{i=1}^{3} \sum_{j=1}^{3} x_{i} x_{j} B_{i j}(T),
$$

whereas the second virial coefficients $B_{i j}$ are evaluated fully quantum-mechanically for the different statistics using two contributions $B_{\text {direct }}$ and $B_{\text {exch }}$ [8]. In the Boltzmann statistics (B) the second virial coefficient is given as

$$
B_{\mathrm{B}}=B_{\text {direct }},
$$

whereas for particles with spin quantum number $s$ according to the Bose-Einstein (BE) or to the Fermi-Dirac (FD) statistics holds:

$$
\begin{aligned}
& B_{\mathrm{BE}}=B_{\text {direct }}+B_{\text {exch }}, \\
& B_{\mathrm{FD}}=B_{\text {direct }}-B_{\text {exch }} .
\end{aligned}
$$

$B_{\text {direct }}$ and $B_{\text {exch }}$ result from summations over the angular momentum quantum number $l$, either over only the even $l$ values or over only the odd $l$ values:

$$
\begin{aligned}
B_{\text {direct }} & =B_{\text {even }}+B_{\text {odd }} \\
B_{\text {exch }} & =\left(\frac{1}{2 s+1}\right)\left(B_{\text {even }}-B_{\text {odd }}-\frac{N_{\mathrm{A}} \Lambda^{3}}{16}\right) .
\end{aligned}
$$

The spin quantum numbers $s$ and the statistics have already been given in Section 2.1 for the six interacting systems composed of the three neon isotopes. The summations over $l$ are represented by:

$$
\begin{aligned}
B(T)= & -\frac{N_{\mathrm{A}} \Lambda^{3}}{2}\left[\sum_{n=0}^{n_{\max } l_{\max }(n)} \sum_{l}^{\infty}(2 l+1)\left(\mathrm{e}^{-\beta E_{n l}^{-}}-1\right)\right. \\
& \left.+\int_{0}^{\infty} \sum_{l}^{\infty}(2 l+1) \frac{\delta_{l}(E)}{\pi} \mathrm{e}^{-\beta E} \mathrm{~d}(\beta E)\right] .
\end{aligned}
$$

Here $\Lambda$ is the thermal wave length:

$$
\Lambda=\left(\frac{h^{2} \beta}{2 \pi \mu}\right)^{\frac{1}{2}}, \quad \beta=\frac{1}{k_{\mathrm{B}} T},
$$

whereas $h$ is Planck's constant and $N_{\mathrm{A}}$ is Avogadro's number. The first term of Eq. (8) corresponds to the contribution of the bound states, where $E_{n l}^{-}$is the nega- 


\section{Mys}

tive eigenvalue of the $n$th vibrational state with the angular-momentum quantum number $l$ which is obtained from the solution of the Schrödinger equation for the radial factor wave functions $\psi_{l}(R)$. Some bound states corresponding to the rotational levels for the three vibrational states of the ground electronic state of the ${ }^{20} \mathrm{Ne}-{ }^{20} \mathrm{Ne}$ and ${ }^{20} \mathrm{Ne}-{ }^{22} \mathrm{Ne}$ dimers were listed for the neon-neon interatomic potential under discussion in Tables 4 and 5 of our paper I [3]. The bound states contribution is particularly of importance at low temperatures. The second term of Eq. (8) is the essential contribution at medium and higher temperatures and is related to the scattering resulting from binary collisions and to the phase shifts $\delta_{l}(E)$. Due to the fact that the sum over $l$ and the integral in Eq. (8) have limits from 0 to $\infty$, serious errors in the computation may occur when truncated inadequately. Therefore, it was verified that the energies for which the evaluation was performed and particularly the number of the phase shifts were chosen large enough.

In the second variant naturally occurring neon is considered as a pure gas consisting of atoms with the average relative atomic mass 20.1797 . Then the second virial coefficient $B(T)$ is derived as the sum of a classical contribution as well as of first-order, second-order, and third-order quantum corrections [9]:

$$
B(T)=B_{\mathrm{cl}}(T)+\lambda B_{\mathrm{qm}, 1}(T)+\lambda^{2} B_{\mathrm{qm}, 2}(T)+\lambda^{3} B_{\mathrm{qm}, 3}(T)+\cdots
$$

with $\lambda=\frac{\hbar^{2} \beta}{12 m}$ and $\hbar=\frac{h}{2 \pi}$. The individual summands are given as:

$$
\begin{aligned}
B_{\mathrm{cl}}(T) & =-2 \pi N_{\mathrm{A}} \int_{0}^{\infty}\left[\mathrm{e}^{-\beta V(R)}-1\right] R^{2} \mathrm{~d} R \\
B_{\mathrm{qm}, 1}(T) & =2 \pi N_{\mathrm{A}} \int_{0}^{\infty}\left[\beta V^{\prime}(R)\right]^{2} \mathrm{e}^{-\beta V(R)} R^{2} \mathrm{~d} R \\
B_{\mathrm{qm}, 2}(T) & =-2 \pi N_{\mathrm{A}} \int_{0}^{\infty}\left\{\frac{6}{5}\left[\beta V^{\prime \prime}(R)\right]^{2}+\frac{12}{5 R^{2}}\left[\beta V^{\prime}(R)\right]^{2}+\frac{4}{3 R}\left[\beta V^{\prime}(R)\right]^{3}\right. \\
& \left.-\frac{1}{6}\left[\beta V^{\prime}(R)\right]^{4}\right\} \mathrm{e}^{-\beta V(R)} R^{2} \mathrm{~d} R \\
B_{\mathrm{qm}, 3}(T) & =2 \pi N_{\mathrm{A}} \int_{0}^{\infty}\left\{\frac{36}{35}\left[\beta V^{\prime \prime \prime}(R)\right]^{2}+\frac{216}{35 R^{2}}\left[\beta V^{\prime \prime}(R)\right]^{2}+\frac{24}{21}\left[\beta V^{\prime \prime}(R)\right]^{3}\right. \\
+\frac{24}{5 R}\left[\beta V^{\prime}(R)\right]\left[\beta V^{\prime \prime}(R)\right]^{2}+\frac{288}{315 R^{3}}\left[\beta V^{\prime}(R)\right]^{3}-\frac{6}{5}\left[\beta V^{\prime}(R)\right]^{2}\left[\beta V^{\prime \prime}(R)\right]^{2} & (13) \\
\left.-\frac{2}{15 R^{2}}\left[\beta V^{\prime}(R)\right]^{4}-\frac{2}{5 R}\left[\beta V^{\prime}(R)\right]^{5}+\frac{1}{30}\left[\beta V^{\prime}(R)\right]^{6}\right\} \mathrm{e}^{-\beta V(R)} R^{2} \mathrm{~d} R & (1
\end{aligned}
$$

For the evaluation of the third pressure virial coefficient $C(T)$, naturally occurring neon is again assumed to be a pure gas composed of atoms with the same 
mass. Furthermore, $C(T)$ is calculated as a sum of three contributions $[10,11]$, one term for the pairwise additivity of the two-body interatomic potentials $C_{\text {add }}$, an extra genuine term $C_{\text {non-add }}$ for the non-additivity $\Delta V_{3}\left(R_{12}, R_{13}, R_{23}\right)$ of the three-body interatomic interaction potential $V_{3}\left(R_{12}, R_{13}, R_{23}\right)$, and a first-order correction term for the quantum effects $C_{\mathrm{qm}, 1}$ :

$$
C(T)=C_{\text {add }}(T)+C_{\text {non-add }}(T)+\lambda C_{\mathrm{qm}, 1}(T)+\cdots
$$

The formulas for the three contributions have already been given in our paper on helium [1] so that it is not necessary to repeat the details. Here the only difference consists in that $\lambda=\frac{\hbar^{2} \beta}{12 m}$ is separated in the first-order correction term of the quantum effects $C_{\mathrm{qm}, 1}$. The non-additivity contribution $\Delta V_{3}\left(R_{12}, R_{13}, R_{23}\right)$ to the three-body potential is again approximated by the Axilrod-Teller triple-dipole potential term $[12,13]$, in which the non-additivity coefficient of the triple-dipole term calculated for neon by Kumar and Meath [14], $C_{9}=1.228 \times 10^{-5} \mathrm{~K}(\mathrm{~nm})^{9}$, is used.

\subsection{Calculation of the transport properties}

Different alternative ways were used to determine the transport properties of naturally occurring neon as a function of temperature $T$. In the first variant $\eta(T)$ and $\lambda(T)$ are evaluated quantum-mechanically and approximately like that of a dilutegas mixture in the limit of zero density composed of the three neon isotopes. In the first-order approximation of the kinetic theory the viscosity is formulated as:

$$
\begin{aligned}
& {\left[\eta_{\text {mix }}\right]_{1}=\frac{\left|\begin{array}{cccc}
H_{11} & H_{12} & H_{13} & x_{1} \\
H_{21} & H_{22} & H_{23} & x_{2} \\
H_{31} & H_{32} & H_{33} & x_{3} \\
x_{1} & x_{2} & x_{3} & 0
\end{array}\right|}{\left|\begin{array}{lll}
H_{11} & H_{12} & H_{13} \\
H_{21} & H_{22} & H_{23} \\
H_{31} & H_{32} & H_{33}
\end{array}\right|}} \\
& H_{i j}=2 x_{i} m_{i} \sum_{k=1}^{3} \frac{x_{k} m_{k}}{\left[\eta_{i k}\right]_{1}\left(m_{i}+m_{k}\right)^{2}}\left[\frac{5}{3 A_{i k}}\left(\delta_{i j}-\delta_{j k}\right)+\frac{m_{k}}{m_{i}} \delta_{i j}+\delta_{j k}\right] \text {, } \\
& {\left[\eta_{i j}\right]_{1}=\frac{5}{16} \frac{\left(2 \pi \mu_{i j} k_{\mathrm{B}} T\right)^{1 / 2}}{\Omega_{i j}^{(2,2)}(T)},} \\
& A_{i k}=\frac{\Omega_{i k}^{(2,2)}(T)}{\Omega_{i k}^{(1,1)}(T)}, \quad \mu_{i j}=\frac{m_{i} m_{j}}{m_{i}+m_{j}} .
\end{aligned}
$$




\section{Molec}

The symbol $\left[\eta_{i j}\right]_{1}$ represents the first-order approximation of the viscosity characterizing the interaction between a binary pair $i-j$. Here all different $\left[\eta_{i j}\right]_{1}$ are given in terms of collision integrals $\Omega_{i j}^{(2,2)}(T)$ which have to be evaluated quantummechanically (see below). $\delta_{i j}$ is the Kronecker symbol, $A_{i k}$ corresponds to the ratio of two collision integrals, whereas $\mu_{i j}$ is the reduced mass of the interacting pair.

In analogy to Eq. (16) an equation in which the elements $H_{i j}$ are replaced by elements $L_{i j}$ is applied for the thermal conductivity of a dilute gas mixture in its first-order approximation. The elements $L_{i j}$ are expressed as:

$$
\begin{aligned}
L_{i j}= & x_{i} m_{i} \sum_{k=1}^{3} \frac{x_{k} m_{k}}{\left[\lambda_{i k}\right]_{1}\left(m_{i}+m_{k}\right)^{2}}\left\{\frac{5}{8 A_{i k}^{*}}\left[\left(6 \frac{m_{i}}{m_{k}}+5 \frac{m_{k}}{m_{i}}\right) \delta_{i j}-11 \delta_{j k}\right]\right. \\
& \left.-\frac{3 B_{i k}}{2 A_{i k}}\left(\frac{m_{k}}{m_{i}} \delta_{i j}-\delta_{j k}\right)+2\left(\delta_{i j}+\delta_{j k}\right)\right\} \\
{\left[\lambda_{i j}\right]_{1}=} & \frac{75}{64} \frac{\left(2 \pi \mu_{i j} k_{\mathrm{B}}^{3} T\right)^{1 / 2}}{2 \mu_{i j} \Omega_{i j}^{(2,2)}(T)} \\
B_{i k}= & \frac{5 \Omega_{i k}^{(1,2)}(T)-4 \Omega_{i k}^{(1,3)}(T)}{\Omega_{i k}^{(1,1)}(T)} .
\end{aligned}
$$

Here $\left[\lambda_{i j}\right]_{1}$ is the first-order approximation of the thermal conductivity related to the interaction between a binary pair $i-j$ and again given in terms of the $\Omega_{i j}^{(2,2)}(T)$ collision integrals. $B_{i k}$ represents a relation between different collision integrals.

In principle, exact calculations require higher-order approximations of the kinetic theory. Therefore, we used fifth-order approximations in the case of the transport properties of helium [1], but the calculations for ${ }^{3} \mathrm{He}$ and ${ }^{4} \mathrm{He}$ concerned a pure gas each. Unfortunately, approximations of such a high order are not available for mixtures so that we were forced to choose any other reasonable way for the higher-order calculations of the transport properties of naturally occurring neon and tested thus two possibilities. On the one hand, the individual viscosity and thermal conductivity coefficients $\eta_{i j}$ and $\lambda_{i j}$ for the binary pairs with like and unlike interactions are calculated up to the fifth-order approximation according to:

$$
\begin{aligned}
{\left[\eta_{i j}\right]_{5} } & =\frac{5}{16} \frac{\left(2 \pi \mu_{i j} k_{\mathrm{B}} T\right)^{1 / 2}}{\Omega_{i j}^{(2,2)}(T)} f_{\eta, i j}^{(5)} \\
{\left[\lambda_{i j}\right]_{5} } & =\frac{75}{64} \frac{\left(2 \pi \mu_{i j} k_{\mathrm{B}}^{3} T\right)^{1 / 2}}{2 \mu_{i j} \Omega_{i j}^{(2,2)}(T)} f_{\lambda, i j}^{(5)} .
\end{aligned}
$$

Here $f_{\eta, i j}^{(5)}$ and $f_{\lambda, i j}^{(5)}$ represent the correction factors for the fifth-order approximations of the kinetic theory. The resulting values for $\left[\eta_{i j}\right]_{5}$ and $\left[\lambda_{i j}\right]_{5}$ are then used in the first-order approximation for the mixture viscosity represented by Eq. (16) as well as in the corresponding relation for the thermal conductivity. On the other 
hand, the values of the first-order approximations $\left[\eta_{\text {mix }}\right]_{1}[$ Eq.(16) $]$ and $\left[\lambda_{\text {mix }}\right]_{1}$ are corrected for the fifth-order approximations by means of correction factors $f_{\eta}^{(5)}$ and $f_{\lambda}^{(5)}$. These are derived using collision integrals which are also determined quantum-mechanically for a pure neon gas consisting of atoms with the average relative atomic mass 20.1797 and following the Bose-Einstein statistics for ${ }^{20} \mathrm{Ne}-$ ${ }^{20} \mathrm{Ne}$. For the calculations of $f_{\eta, i j}^{(5)}$ and $f_{\lambda, i j}^{(5)}$ as well as $f_{\eta}^{(5)}$ and $f_{\lambda}^{(5)}$ we used explicit expressions and computer programs provided by Viehland et al. [15]. According to our calculations the effect of the fifth-order corrections to the viscosity and to the thermal conductivity compared with the fourth-order corrections is below $\pm 0.01 \%$.

Expressions for the quantum cross sections $Q^{(m)}(E)$ and for the quantum collision integrals $\Omega^{(m, s)}(T)$ needed in the different approximations of the solution of the Boltzmann equation were derived by Meeks et al. [16]. They were again collected in our paper for helium [1] for particles with spin $s$ according to the Bose-Einstein (BE) or to the Fermi-Dirac (FD) statistics as well as for the Boltzmann statistics. The formulas for the different $Q^{(m)}$ are related to sums over the phase shifts $\delta_{l}$, either over only the odd $l$ values or over only the even $l$ values, but also over complete sums (Boltzmann statistics). The quantum collision integrals $\Omega^{(m, s)}(T)$ result from the quantum cross sections $Q^{(m)}(E)$ according to:

$$
\Omega^{(m, s)}(T)=\frac{4 \pi \hbar^{2}}{2 \mu k_{\mathrm{B}} T(s+1) !} \int_{0}^{\infty} Q^{(m)}(E) \mathrm{e}^{-\beta E}(\beta E)^{s} \mathrm{~d}(\beta E) .
$$

In a second variant the viscosity of neon was determined classically for the firstorder and the fifth-order approximations in order to examine whether a quantummechanical calculation is actually needed to achieve highly accurate values for the transport properties of neon in the zero-density limit. For this purpose the usual formulations for monatomics [17] were used, whereas neon was again considered to be a pure gas with the average relative atomic mass already given.

\section{Comparison with experimental data and values for other interatomic potentials}

\subsection{Second and third pressure virial coefficients}

The quantum-mechanical calculation of the second pressure virial coefficient requires the determination of the existing bound states. For this purpose the Level 7.7 program of LeRoy [18] was used. As already mentioned in Section 2.2, the bound states of the ${ }^{20} \mathrm{Ne}-{ }^{20} \mathrm{Ne}$ and ${ }^{20} \mathrm{Ne}-{ }^{22} \mathrm{Ne}$ dimers were listed in our paper I [3] in which they were compared with the experimental rovibrational spectra by Wüest and Merkt [5].

In figure 1 the values calculated fully quantum-mechanically for the interatomic potential of the present paper are opposed to those resulting from quantum cor- 


\section{Molecu}

rections of increasing order added to the classical contribution. The figure illustrates that the classical contribution alone is completely insufficient to describe adequately the second pressure virial coefficient. The agreement between both ways of calculation improves with the quantum corrections included what becomes obvious particularly at low temperatures. To obtain close agreement even at the lowest temperatures, the third-order quantum correction is needed.

There exists only a limited number of experimental data for second and third pressure virial coefficients of neon in the literature compared with those of common gases like argon and nitrogen as well as with those of helium. Furthermore, it is to point out that experimental data for the third pressure virial coefficient are not independent of the values for the second pressure virial coefficient derived from the same experiments. Hence third pressure virial coefficients combined with second ones are included in the comparison. Second and third pressure virial coefficients were determined by Holborn and Otto [19], Nicholson and Schneider [20], Michels et al. [21], and Gibbons [22] from isothermal measurements of volume (and density, respectively) and pressure. Vogl and Hall [23] used a Burnett apparatus to derive isothermal compression factors and to obtain finally second and third pressure virial coefficients. Unfortunately, in none of these papers an error propagation analysis or uncertainties of the second and third pressure virial coefficients adequately deduced from the experiments were reported.

The experimental $B$ data are compared with the values calculated fully quantummechanically for the neon-neon interatomic potential of the present paper in figure 2 , in which the absolute deviations $B_{\exp }-B_{\text {cal(pres) }}$ are displayed. The figure demonstrates a very good agreement for the excellent data by Michels et al. [21] at medium temperatures. A good agreement is also found for the data by Nicholson and Schneider [20] up to high temperatures of 1,000 K. On the contrary, the very old data of Holborn and Otto [19] as well as the more recent but also already 35 years old data of Vogl and Hall [23] are characterized by comparably larger differences to the theoretically calculated values. The data of Gibbons [22] determined at low temperatures show partly large deviations, but agree partly very well. In figure 2 our calculated values are additionally compared with the values calculated for the interatomic potentials by Aziz and Slaman [7], Cybulski and Toczylowski [6], and Wüest and Merkt [5]. The differences $B_{\text {cal(lit) }}-B_{\text {cal(pres) }}$ derived for the different interatomic potentials increase to low temperatures, where the values derived from the potentials by Aziz and Slaman [7] and Wüest and Merkt [5] are too small and the values resulting from the potential by Cybulski and Toczylowski [6] are too large. At medium and higher temperatures the $B_{\text {cal }}$ values for all four potentials do not differ much so that the second pressure virial coefficient is not suitable to distinguish between the different interatomic potentials.

In figure 3 a comparison between experimental data of the third pressure virial 
coefficient of neon and values calculated for the new interatomic potential is shown. The figure elucidates that good agreement of the experimental data by Michels et al. [21] and by Nicholson and Schneider [20] at medium temperatures and of the data by Gibbons [22] at low temperatures with the calculated values is only achieved in the case of the complete sum of the contributions for the pairwise additivity $C_{\text {add }}$, for the non-additivity of the three-body interatomic interactions according to Axilrod and Teller $C_{\text {non-add }}$, and for the first-order quantum-mechanical correction $\lambda C_{\mathrm{qm}, 1}$. The experimental data by Holborn and Otto [19] as well as Vogl and Hall [23] possess again larger differences to the calculated values. The comparison makes evident that the calculation procedure for the third pressure virial coefficient predicts very good values.

It is to be stressed that the calculated values for the second and the third pressure virial coefficients are more reliable than the experimental data at low and high temperatures.

\section{$3.2 \quad$ Viscosity}

First, the results of the different alternative ways of the calculation of the transport properties of naturally occurring neon are compared. In this context it is sufficient to consider only the viscosity, since the effects are the same for the thermal conductivity. Figure 4 illustrates the relative differences between viscosity values derived for the different approximation procedures and the viscosity values obtained from the quantum-mechanical calculation up to the fifth-order approximation for the individual $\left[\eta_{i j}\right]_{\mathrm{qm}, 5}$ within the first-order formulation of $\left[\eta_{\text {mix }}\right]_{1}$ (see Section 2.3). The figure makes evident that the first-order approximation of the classical calculation leads to values which are nearly $1 \%$ too small in the complete temperature range except at the lowest temperatures. The agreement improves when the fifth-order approximation of the classical evaluation is applied. But even for this high-order approximation it becomes obvious that the classical evaluation is not appropriate with regard to highly accurate values. Thus the deviations from the results for the quantum-mechanical calculation of the same fifth-order approximation amount to $-0.1 \%$ at room temperature increasing up to $-1.1 \%$ at about $60 \mathrm{~K}$. On the other hand, the first-order approximation of the quantum-mechanical calculation for a dilute-gas mixture composed of the three neon isotopes according to $\left[\eta_{\text {mix }}\right]_{\mathrm{qm}, 1}$ is not adequate, too. The differences are approximately $-0.7 \%$ at most temperatures and decrease to zero at the lowest temperatures. Further it is to note that there are only differences of $< \pm 0.0004 \%$ (not visible in figure 4) between the results for the two ways to correct the first-order approximation $\left[\eta_{\text {mix }}\right]_{\mathrm{qm}, 1}$ to an appropriate fifth-order approximation of the quantum-mechanical determination. In the following the comparisons with experimental data are performed with values resulting for the fifth-order approximation of the individual $\left[\eta_{i j}\right]_{\mathrm{qm}, 5}$ and $\left[\lambda_{i j}\right]_{\mathrm{qm}, 5}$ within the 


\section{Molecu}

first-order formulations of $\left[\eta_{\mathrm{mix}}\right]_{1}$ and $\left[\lambda_{\mathrm{mix}}\right]_{1}$.

With regard to the transport properties it is to consider that most measurements at low densities were performed at atmospheric pressure, whereas the theoretical calculations are valid for the limit of zero density. Hence the initial density dependence of the experimental data would have to be taken into account. However apart from the very low temperatures near to the normal boiling point of neon, the effect of the initial density dependence on the transport properties concerning the change in density from that at atmospheric pressure to zero density is comparably small $(<0.1 \%)$ for all other temperatures. Furthermore, the experimental uncertainty is distinctly increased at low temperatures.

In our paper concerning the thermophysical standard values for low-density helium [1] we argued that it is difficult to perform genuine absolute measurements of the gas viscosity with an uncertainty $< \pm 0.1 \%$, even at room temperature. The same complex of problems is illustrated in figure 5 in which the best experimental data for neon near to ambient temperature are characterized by error bars for the uncertainties, given by the authors themselves, and are compared with the viscosity values calculated quantum-mechanically. For helium we demonstrated that the measurements with an oscillating-disk viscometer by Kestin and Leidenfrost [24], approved as one of the most accurate and additionally one of the few absolute measurements on gases, can only partly be considered as absolute ones, since they were finally adjusted to a value for the viscosity of air at $293.15 \mathrm{~K}$ and at atmospheric pressure determined by Bearden [31] in an absolute measurement with a rotatingcylinder viscometer. Thus the viscosity value of Kestin and Leidenfrost for neon at $20^{\circ} \mathrm{C}$ (uncertainty: $\pm 0.05 \%$ ) shown in figure 5 corresponds as well to a relative measurement, whereas the genuine absolute measurement is that of Bearden on air. Measurements by Kestin and Nagashima [25], performed in a nearly analogous procedure, led to values which are $0.15-0.3 \%$ higher than those of Kestin and Leidenfrost [24], but also by the same percentage higher than further data obtained in relative measurements of the same research group by DiPippo et al. [26] as well as a best estimate reported by Kestin et al. [27] in 1972 as a result of their measurements in foregoing years. This shows that there were sometimes surprisingly large differences in the results of the measurements of this group. Nevertheless, the results of the most reliable measurements by Kestin and co-workers at ambient temperature are characterized by a tendency to values increased by $+0.1 \%$ compared to the calculated values of the present paper. The same findings concerning the measurements by Kestin and co-workers were observed in the case of the values derived from our interatomic helium potential. As a consequence, the measurements on helium by Vogel [29] (uncertainty: $\pm 0.15 \%$ at room temperature) performed with an all-quartz oscillating-disk viscometer in a relative manner using a viscometer constant derived from the best estimate by Kestin et al. should be 
affected by the same impact. Therefore, the viscometer of Vogel was recalibrated with the new helium standard for a rehandled evaluation of the measurements on helium [1] and on neon, too. The influence of the recalibration on the results of Vogel for neon is additionally demonstrated in figure 5. A value at $298.15 \mathrm{~K}$ resulting from the fitting function given by Vogel [29] deviates from the value calculated for the interatomic neon potential of the present paper by $+0.18 \%$. On the contrary, the direct experimental data of the measurement series by Vogel at room temperature show only differences of $+0.04 \%$ and $+0.08 \%$ after the recalibration.

Furthermore, the absolute measurements by Flynn et al. [28] performed with a capillary viscometer led to a datum at $293.15 \mathrm{~K}$ differing only by $+0.01 \%$ from the theoretically calculated value (uncertainties: $\pm 0.1 \%$ ). Recently, Evers et al. [30] utilized a rotating-cylinder viscometer for absolute measurements (uncertainty: $\pm 0.15 \%)$ on several gases at different temperatures and pressures. Their result for neon at $298.15 \mathrm{~K}$ deviates from our calculated value by $-0.12 \%$. In conclusion, the comparison makes evident that the best experimental data at room temperature are characterized by an uncertainty of $\pm(0.1$ to 0.15$) \%$ and that they agree within this limit with the values calculated for the interatomic neon potential of the present paper.

The situation deteriorates to the disadvantage of the experiment, if the measurements were not performed at ambient temperature. In figure 6, experimental data at low and medium temperatures between $20 \mathrm{~K}$ and $373 \mathrm{~K}$ are compared with the calculated values. Error bars for one or two (in the case that the uncertainty changes with temperature) values of each data set are additionally plotted. The figure demonstrates that excellent agreement within $\pm 0.1 \%$ exists only for the absolute measurement by Evers et al. [30] at $348 \mathrm{~K}$ and that the results of the absolute measurements by Flynn et al. [28] are adequately consistent within $\pm 0.3 \%$. The other data were determined by relative measurements, which are not only affected by the usual measurement errors, but also by the values used for the calibration. Johnston and Grilly [32] and Rietveld et al. [34] (both using oscillating-disk viscometers) as well as Clarke and Smith [35] (capillary viscometer) based their measurements on reasonable values for air, helium, and nitrogen at ambient temperature and achieved results with deviations up to $-2 \%,+4 \%$, and $+1 \%$. These data are not suitable to judge the appropriateness of any interatomic neon potential. On the other hand, the measurements by Coremans et al. [33] carried out with an oscillating-disk viscometer, which was calibrated using a very old viscosity value for ${ }^{4} \mathrm{He}$ at $20 \mathrm{~K}$ reported by Kamerlingh Onnes and Weber [36], yielded values characterized by positive deviations up to $6 \%$ from the quantum-mechanically calculated values. These results were improved for the purposes of this paper by a recalibration with a value for ${ }^{4} \mathrm{He}$ at $20 \mathrm{~K}$ taken from our new helium standard [1]. Figure 6 makes obvious that the corrected data advanced after this correction 


\section{Molecul}

partly in close agreement.

Figure 7 illustrates the analogous comparison at higher temperatures. The figure reveals a surprisingly large scattering of about $\pm 0.3 \%$ in the data from different papers by Kestin and his research group [27,37,38] (the same order of magnitude as the uncertainty) and additionally a systematic trend to higher values with increasing temperature combined with again decreasing values at the highest temperatures. In this connection it is to note that all measurements by Kestin and his co-workers with the oscillating-disk viscometer by Di Pippo et al. [41] are affected by a temperature measurement error with thermocouples explained by Vogel et al. [42]. Figure 7 makes also evident that the data by Vogel [29], originally fitted to his experiments which were based on a calibration with the best estimate value at room temperature by Kestin et al. [27], deviate by about $+0.2 \%$ from the quantum-mechanically calculated values of this paper. After a recalibration of the measurement series on neon by means of the new helium standard [1] at room temperature, the corrected experimental data do only deviate by less than $+0.1 \%$ on average from the theoretical values for the new neon potential in the complete temperature range of the measurements. This demonstrates that the measurements by Vogel with his all-quartz oscillating-disk viscometer represent the best experiments in this temperature range. The comparison concerning the experimental data by Dawe and Smith [39] and by Guevara and Stensland [40], which result from relative measurements with capillary viscometers based on a reasonable calibration at room temperature, shows that these data should be influenced by systematic errors. Lastly it is concluded that the theoretical determination of viscosity values is to be preferred to experiments at these high temperatures.

Figures 5, 6, and 7 include once again a comparison with the values derived classically using the fifth-order approximation. The results of the classical calculation deviate by about $-0.1 \%$ from those of the quantum-mechanical computation at ambient and higher temperatures. At lower temperatures the deviations are distinctly increased. Figure 5 elucidates further that at room temperature the results of the quantum-mechanical calculations for the potentials by Aziz and Slaman [7] and by Cybulski and Toczylowski [6] (both $> \pm 0.2 \%$ ) and particularly by Wüest and Merkt [5] ( $-0.7 \%$, not observable in the figure) do not match the best experimental data as well as the calculated values for the potential of the present paper within $\pm 0.1 \%$. Figure 6 and 7 demonstrate that the best experimental data allow to distinguish between the different potentials proposed for neon. The values resulting from the potentials by Aziz and Slaman [7] and by Cybulski and Toczylowski [6] and particularly by Wüest and Merkt [5] are characterized by differences from the transport data that are distinctly larger than the experimental uncertainties. Here it is to point to the differences for the values determined with the potential proposed by Wüest and Merkt [5]. They arise with increasing temperature due to the 
fact that the rovibrational spectra used by Wüest and Merkt are sensitive to the shape of the potential well, but not to the repulsive part of the potential to which the transport properties are particularly sensitive.

\subsection{Thermal conductivity}

The uncertainty of measurements of the thermal conductivity is inferior to that of viscosity measurements due to different experimental difficulties, whereas the most accurate data can be obtained with the transient hot-wire technique, but essentially restricted to ambient temperature. This is demonstrated in figure 8 , in which experimental data for neon at low and medium temperatures are compared with the values calculated quantum-mechanically. Here the experimental data are again, when available, characterized by error bars according to the uncertainties given by the experimenters themselves. The data by Kestin et al. [44] and Assael et al. [45], each gained with the transient hot-wire technique at room temperature, deviate from the calculated values by $<-0.1 \%$ and $<+0.2 \%$. These differences are lower than the experimental uncertainties $( \pm 0.3 \%$ and $\pm 0.2 \%)$. Although the data by Haarman [43] are characterized by larger deviations $(-0.3 \%$ to $-0.4 \%)$, the temperature function of these transient hot-wire data between $328 \mathrm{~K}$ and $468 \mathrm{~K}$ corresponds closely to that of the calculated values. On the contrary, the temperature function of the data by Millat et al. [46] shows an awkward behaviour so that these data are not useful with regard to the assessment of the values calculated for the different interatomic potentials of neon. But figure 8 makes also evident that the deviations of the experimental data of Hemminger [47], derived from measurements with a guarded parallel-plate apparatus and carefully corrected for impurities caused by desorbed air, are within $-0.35 \%$ and $-0.6 \%$; this means their temperature function and that of the calculated values are pretty much consistent from room temperature up to $470 \mathrm{~K}$.

Experimental data determined with the common steady-state hot-wire technique often affected by convection are checked against the quantum-mechanically calculated values in figure 8, too. Differences of only $< \pm 0.4 \%$ are found for the very old experimental datum by Weber [48] at $273 \mathrm{~K}$ and also for a value by Kannuluik and Carman [49] at the same temperature. But for the complete temperature range of the measurements of Kannuluik and Carman between $90 \mathrm{~K}$ and $580 \mathrm{~K}$ the deviations increase up to $-3 \%$. On the other hand, the smoothed experimental values by Nesterov and Sudnik [52] between $90 \mathrm{~K}$ and ambient temperature created with the same technique are characterized by comparably small differences between $-0.1 \%$ and $-0.7 \%$, with the best agreement at low temperatures. Further it becomes evident from this figure that the experimental data by Keyes [50] determined with the concentric-cylinder method (differences between $-1 \%$ and $-1.5 \%$ ) and those of Sengers et al. [51] obtained with a parallel-plate apparatus (differences 


\section{Molecul}

\section{Summary and conclusions}

A new interatomic potential for neon derived from quantum-mechanical $a b$ initio computations [3] was utilized to calculate the second and third pressure virial, the viscosity and the thermal conductivity coefficients for dilute neon gas in its natural isotopic composition in the temperature range from $25 \mathrm{~K}$ to $10,000 \mathrm{~K}$. For the second virial coefficient and for the transport properties fully quantum-mechanical calculations were performed with neon treated as an isotopic mixture, whereas for the third virial coefficient a classical mechanical evaluation with a quantum correction using the average mass of the isotopic mixture was applied. The comparison with available experimental data makes evident that the calculated thermophysical properties are as accurate as the best experimental data at room temperature and more accurate at temperatures above and below room temperature. The deviations between the results from the different potentials for all calculated properties increase at the lowest temperatures. 
The viscosity values around ambient temperature derived theoretically with the interatomic potential of this paper are characterized by deviations smaller than $\pm 0.1 \%$ compared to the best experimental data, whereas the results obtained from the potential energy curves by Cybulski and Toczylowski, by Aziz and Slaman [7], and by Wüest and Merkt [5] show larger deviations. We estimate summarily the uncertainties of the calculated transport properties resulting from our new potential to be about $\pm 0.1 \%$ except at the lowest temperatures. It is to be stressed that this uncertainty is much below the experimental uncertainties at low as well as at high temperatures. All calculated data (see table A in the appendix) can be applied as standards values for the complete temperature range. 


\section{References}

[1] E. Bich, R. Hellmann, and E. Vogel, Mol. Phys. 105, 3035 (2007).

[2] R. Hellmann, E. Bich, and E. Vogel, Mol. Phys. 105, 3013 (2007).

[3] R. Hellmann, E. Bich, and E. Vogel, Mol. Phys. in press.

[4] K. T. Tang and J. P. Toennies, J. Chem. Phys. 80, 3726 (1984).

[5] A. Wüest and F. Merkt, J. Chem. Phys. 118, 8807 (2003).

[6] S. M. Cybulski and R. R. Toczylowski, J. Chem. Phys. 111, 10520 (1999).

[7] R. A. Aziz and M. J. Slaman, Chem. Phys. 130, 187 (1989).

[8] M. E. Boyd, S. Y. Larsen, and J. E. Kilpatrick, J. Chem. Phys. 50, 4034 (1969).

[9] E. A. Mason and T. H. Spurling, The Virial Equation of State (Pergamon, Oxford, 1969).

[10] S. Kim and D. Henderson, Proc. Nat. Acad. Sci., Wash., 55, 705 (1966).

[11] K. Lucas, Angewandte Statistische Thermodynamik (Springer, Berlin, 1986).

[12] B. M. Axilrod and E. Teller, J. Chem. Phys. 11, 299 (1943).

[13] B. M. Axilrod, J. Chem. Phys. 19, 719 (1951).

[14] A. Kumar and W. J. Meath, Mol. Phys. 54, 823 (1985).

[15] L. A. Viehland, A. R. Janzen, and R. A. Aziz, J. Chem. Phys. 102, 5444 (1995).

[16] F. R. Meeks, T. J. Cleland, K. E. Hutchinson, and W. L. Taylor, J. Chem. Phys. 100, 3813 (1994).

[17] G. C. Maitland, M. Rigby, E. B. Smith, and W. A. Wakeham, Intermolecular Forces. Their Origin and Determination (Clarendon Press, Oxford, 1987).

[18] R. J. LeRoy, LEVEL 7.7. A Computer Program for Solving the Radial Schrödinger Equation for Bound and Quasibound Levels, University of Waterloo, Chemical Physics Research Report CP-661 (Waterloo, Ontario, Canada, 2005).

[19] L. Holborn and J. Otto, Z. Phys. 33, 1 (1925); 38, 359 (1926).

[20] G. A. Nicholson and W. G. Schneider, Can. J. Chem. 33, 589 (1955).

[21] A. Michels, T. Wassenaar, and P. Louwerse, Physica 26, 539 (1960).

[22] R. M. Gibbons, Cryogenics 9, 251 (1969).

[23] W. F. Vogl and K. R. Hall, Physica 59, 529 (1972).

[24] J. Kestin and W. Leidenfrost, Physica 25, 1033 (1959).

[25] J. Kestin and A. Nagashima, J. Chem. Phys. 40, 3648 (1964).

[26] R. DiPippo, J. Kestin, and K. Oguchi, J. Chem. Phys. 46, 4758 (1967).

[27] J. Kestin, S. T. Ro, and W. A. Wakeham, J. Chem. Phys. 56, 4119 (1972).

[28] G. P. Flynn, R. V. Hanks, N. A. Lemaire, and J. Ross, J. Chem. Phys. 38, 154 (1963).

[29] E. Vogel, Ber. Bunsenges. Phys. Chem. 88, 997 (1984).

[30] C. Evers, H. W. Lösch, and W. Wagner, Int. J. Thermophys. 23, 1411 (2002).

[31] J. A. Bearden, Phys. Rev. 56, 1023 (1939).

[32] H. L. Johnston and E. R. Grilly, J. Phys. Chem. 46, 948 (1942).

[33] J. M. J. Coremans, A. van Itterbeek, J. J. M. Beenakker, H. F. P. Knaap, and P. Zandbergen, Physica 24, 557 (1958).

[34] A. O. Rietveld, A. van Itterbeek, and C. A. Velds, Physica 25, 205 (1959).

[35] A. G. Clarke and E. B. Smith, J. Chem. Phys. 51, 4156 (1969).

[36] H. Kamerlingh Onnes and S. Weber, Vers. Kon. Acad. Wetenschapen Amsterdam 21, 1385 (1913).

[37] J. M. Hellemans, J. Kestin, and S. T. Ro, Physica 71, 1 (1974).

[38] J. Kestin, H. E. Khalifa, and W. A. Wakeham, Physica A 90, 215 (1978).

[39] R. A. Dawe and E. B. Smith, J. Chem. Phys. 52, 693 (1970).

[40] F. A. Guevara and G. Stensland, Phys. Fluids 14, 746 (1971).

[41] R. DiPippo, J. Kestin and J. H. Whitelaw, Physica 32, 2064 (1966).

[42] E. Vogel, C. Küchenmeister, E. Bich, and A. Laesecke, J. Phys. Chem. Ref. Data 27, 947 (1998).

[43] J. W. Haarman, Amer. Inst. Phys. Conf. Proc. 11, 193 (1973).

[44] J. Kestin, R. Paul, A. A. Clifford, and W. A. Wakeham, Physica A 100, 349 (1980).

[45] M. J. Assael, M. Dix, A. Lucas, and W. A. Wakeham, J. Chem. Soc. Faraday Trans. I 77, 439 (1981).

[46] J. Millat, M. Ross, W. A. Wakeham, and M. Zalaf, Physica A 148, 124 (1988).

[47] W. Hemminger, Int. J. Thermophys 8, 317 (1987).

[48] S. Weber, Ann. Phys. 82, 479 (1927).

[49] W. G. Kannuluik and E. H. Carman, Proc. Phys. Soc. London B 65, 701 (1952).

[50] F. G. Keyes, Trans. ASME 76, 809 (1954).

[51] J. V. Sengers, W. T. Bolk, and C. J. Stigter, Physica 30, 1018 (1964).

[52] N. A. Nesterov and V. M. Sudnik, Inzh.-fiz. Zh. 30, 863 (1976).

[53] V. K. Saxena and S. C. Saxena, J. Chem. Phys. 48, 5662 (1968). 
[54] R. Tufeu, B. LeNeindre, and P. Bury, C. R. Acad. Sci. Paris, B 271, 589 (1970).

[55] G. S. Springer and E. W. Wingeier, J. Chem. Phys. 59, 2747 (1973).

[56] H. Ziebland, Pure Appl. Chem. 53, 1863 (1981).

[57] D. J. Collins and W. A. Menard, Trans. ASME 88, 52 (1966).

[58] J. Maštovský, Report Z-1026/87 (C̆SAV, Ústav Termomechaniky, Prague, 1987). 
Table 1. Potential parameters

\begin{tabular}{llr}
\hline$A$ & $\mathrm{~K}$ & $0.402915058383 \mathrm{E}+08$ \\
$a_{1}$ & $(\mathrm{~nm})^{-1}$ & $-0.428654039586 \mathrm{E}+02$ \\
$a_{2}$ & $(\mathrm{~nm})^{-2}$ & $-0.333818674327 \mathrm{E}+01$ \\
$a_{-1}$ & $\mathrm{~nm}$ & $-0.534644860719 \mathrm{E}-01$ \\
$a_{-2}$ & $(\mathrm{~nm})^{2}$ & $0.501774999419 \mathrm{E}-02$ \\
$b$ & $(\mathrm{~nm})^{-1}$ & $0.492438731676 \mathrm{E}+02$ \\
$C_{6}$ & $\mathrm{~K}(\mathrm{~nm})^{6}$ & $0.440676750157 \mathrm{E}-01$ \\
$C_{8}$ & $\mathrm{~K}(\mathrm{~nm})^{8}$ & $0.164892507701 \mathrm{E}-02$ \\
$C_{10}$ & $\mathrm{~K}(\mathrm{~nm})^{10}$ & $0.790473640524 \mathrm{E}-04$ \\
$C_{12}$ & $\mathrm{~K}(\mathrm{~nm})^{12}$ & $0.485489170103 \mathrm{E}-05$ \\
$C_{14}$ & $\mathrm{~K}(\mathrm{~nm})^{14}$ & $0.382012334054 \mathrm{E}-06$ \\
$C_{16}$ & $\mathrm{~K}(\mathrm{~nm})^{16}$ & $0.385106552963 \mathrm{E}-07$ \\
\hline \multirow{2}{*}{$k_{k_{\mathrm{B}}}$} & $\mathrm{K}$ & \multicolumn{2}{|l}{42.152521} \\
$R_{\varepsilon}$ & $\mathrm{nm}$ & 0.30894556 \\
$\sigma$ & $\mathrm{nm}$ & 0.27612487 \\
\hline & &
\end{tabular}

5

9

10

11

12

13

14

15

16

17

18

19

20

21

22

23

24

25

26

27

28

29

30

31

32

33

34

35

36

37

38

39

40

41

42

43

44

45

46

47

48

49

50

51

52

53

54

55

56

57

58

59 
20

Table 2. Number of calculated phase shifts of neon for some reduced energies

\begin{tabular}{ccc}
\hline$E^{*}$ & Total number & Full QM calculation \\
\hline 0. & 11 & 11 \\
$1 . \times 10^{-4}$ & 14 & 12 \\
$1 . \times 10^{-3}$ & 14 & 12 \\
$1 . \times 10^{-2}$ & 29 & 23 \\
$5 . \times 10^{-2}$ & 62 & 34 \\
$1 . \times 10^{-1}$ & 87 & 41 \\
$5 . \times 10^{-1}$ & 177 & 58 \\
1. & 244 & 67 \\
3. & 511 & 84 \\
5. & 1152 & 93 \\
10. & 1152 & 108 \\
100. & 1152 & 131 \\
$3,200$. & 1351 & 131 \\
\hline
\end{tabular}

19 


\section{Molecu}

Fig. 1 Differences $\Delta B=B_{\mathrm{qm}, \text { full }}-\left[B_{\mathrm{cl}}+\sum \lambda^{i} B_{\mathrm{qm}, i}\right]$ between the fully quantum-mechanically calculated values and the values resulting from the sum of a classical contribution and of different orders of quantum corrections to the second pressure virial coefficient for the new interatomic potential for Ne. Differences related to: $\cdots \ldots \ldots \ldots$ classical contribution $B_{\mathrm{cl}} ;-\cdot-\cdot-\cdot$ sum of classical contribution and of first-order quantum correction $B_{\mathrm{cl}}+\lambda B_{\mathrm{qm}, 1} ;----$ sum of classical contribution as well as of first-order and second-order quantum corrections $B_{\mathrm{cl}}+\lambda B_{\mathrm{qm}, 1}+\lambda^{2} B_{\mathrm{qm}, 2} ;-$ sum of classical contribution as well as of first-order, second-order, and third-order quantum corrections $B_{\mathrm{cl}}+\lambda B_{\mathrm{qm}, 1}+\lambda^{2} B_{\mathrm{qm}, 2}+\lambda^{3} B_{\mathrm{qm}, 3}$.

Fig. 2 Differences $\left(B-B_{\text {cal (pres) }}\right)$ of experimental $\left(B_{\exp }\right)$ and calculated $\left(B_{\text {cal(lit) }}\right)$ second pressure virial coefficients from values $\left(B_{\text {cal(pres })}\right)$ calculated with the new interatomic potential for Ne. Experimental data: $\circ$ Holborn and Otto [19]; $\triangle$ Nicholson and Schneider [20]; $\square$ Michels et al. [21]; $\nabla$ Gibbons [22]; $\diamond$ Vogl and Hall [23]. Calculated values: — potential by Aziz and Slaman [7]; - - - - - $\cdot$ potential by Cybulski and Toczylowski [6]; - - - potential by Wüest and Merkt [5].

Fig. 3 Comparison of experimental data and and of calculated values for the third pressure virial coefficient $C$ derived from the new interatomic potential for Ne. Experimental data: $\circ$ Holborn and Otto [19]; $\triangle$ Nicholson and Schneider [20]; $\square$ Michels et al. [21]; $\nabla$ Gibbons [22]; $\diamond$ Vogl and Hall [23]. Calculated values: ---- classical contribution $C_{\text {add }},-\cdot-$. - c classical and non-additivity contributions $C_{\text {add }}+C_{\text {non-add }}$, sum of classical and non-additivity contributions and of the first-order quantum correction $C_{\mathrm{add}}+C_{\text {non-add }}+\lambda C_{\mathrm{qm}, 1}$.

Fig. 4 Relative deviations $\Delta=100\left(\eta-\eta_{\mathrm{qm}, 5}\right) / \eta_{\mathrm{qm}, 5}$ between viscosity values calculated for different approximation procedures and viscosity values resulting from quantum-mechanical calculations up to the fifth-order approximation for the individual $\left[\eta_{i j}\right]_{\mathrm{qm}, 5}$ within the first-order formulation of $\left[\eta_{\text {mix }}\right]_{1}$ for the new interatomic potential for Ne. Differences related to: $\cdots \cdots \cdots \cdots$ first-order classical calculation $[\eta]_{\mathrm{cl}, 1} ;-\cdot \cdot-\cdot \cdot-$ .. fifth-order classical calculation $[\eta]_{\mathrm{cl}, 5} ;-\cdot-\cdot-\cdot$ first-order quantummechanical calculation $\left[\eta_{\text {mix }}\right]_{\mathrm{qm}, 1}$.

Fig. 5 Relative deviations of experimental and calculated viscosity coefficients from values $\eta_{\text {cal(pres) }}$ calculated quantum-mechanically with the new interatomic potential for Ne near to room temperature. Experimental data with uncertainties characterized by error bars: $\bullet$ Kestin and Leidenfrost [24]; ○ Kestin and Nagashima [25]; $\mathbf{\nabla}$ DiPippo et al. [26]; $\nabla$ Kestin et al. [27], best estimate; - Flynn et al. [28]; $\triangle$ Vogel [29], fit- 
ted value; $\boldsymbol{\Delta}$ Vogel [29], experimental data corrected according to new helium standard; Evers et al. [30]. Calculated values: .......... fifth-order classical calculation $[\eta]_{\mathrm{cl}, 5} ;-$ potential by Aziz and Slaman [7]; $-\cdot-\cdot-\cdot$ potential by Cybulski and Toczylowski [6].

Fig. 6 Relative deviations of experimental and calculated viscosity coefficients from values $\eta_{\text {cal(pres) }}$ calculated with the new interatomic potential for Ne at low and medium temperatures. Experimental data with uncertainties characterized by error bars: $\square$ Johnston and Grilly [32]; ○ Coremans et al. [33]; • Coremans et al. [33], corrected according to new helium standard; $\triangle$ Rietveld et al. [34]; - Flynn et al. [28]; $\diamond$ Clarke and Smith [35]; Evers et al. [30]. Calculated values: .......... fifth-order classical calculation $[\eta]_{\mathrm{cl}, 5}$ - potential by Aziz and Slaman $[7] ;-\cdot-\cdot-\cdot$ potential by Cybulski and Toczylowski $[6] ;--$ - - potential by Wüest and Merkt [5].

Fig. 7 Deviations of experimental and calculated viscosity coefficients from values $\eta_{\text {cal(pres) }}$ calculated with the new interatomic potential for $\mathrm{Ne}$ at higher temperatures. Experimental data with uncertainties characterized by error bars: $\nabla$ Kestin et al. [27], best estimate; ○ Hellemans et al. [37]; $\odot$ Kestin et al. [38]; $\diamond$ Dawe and Smith [39]; $\square$ Guevara and Stensland [40]; $\triangle$ Vogel [29], fitted values; $\Delta$ Vogel [29], experimental data corrected according to new helium standard. Calculated values: ......... fifth-order classical calculation $[\eta]_{\mathrm{cl}, 5} ;-$ potential by Aziz and Slaman [7]; - - - . - p potential by Cybulski and Toczylowski [6]; - - - potential by Wüest and Merkt [5].

Fig. 8 Deviations of experimental and calculated thermal conductivity coefficients from values $\lambda_{\text {cal(pres) }}$ calculated with the new interatomic potential for Ne at low and medium temperatures. Experimental data with uncertainties characterized by error bars: • Haarman [43]; $\boldsymbol{\Delta}$ Kestin et al. [44]; - Assael et al. [45]; $\boldsymbol{\nabla}$ Millat et al. [46]; $\diamond$ Hemminger [47]; ○ Weber [48]; $\triangle$ Kannuluik and Carman [49]; $\square$ Keyes [50]; $\nabla$ Sengers et al. [51]; $\diamond$ Nesterov and Sudnik [52], smoothed values. Calculated values: $\cdots \cdots \cdots \cdots$ fifth-order classical calculation $[\eta]_{\mathrm{cl}, 5} ;$ potential by Aziz and Slaman [7]; $-\cdot-\cdot \cdot \cdot$ potential by Cybulski and Toczylowski [6]; - - - - potential by Wüest and Merkt [5].

Fig. 9 Deviations of experimental and calculated thermal conductivity coefficients from values $\lambda_{\text {cal(pres) }}$ calculated with the new interatomic potential for $\mathrm{Ne}$ at higher temperatures. Experimental data with uncertainties characterized by error bars: ○ Saxena and Saxena [53], smoothed values; $\triangle$ Tufeu et al. [54]; $\square$ Springer and Wingeier [55]; $\nabla$ Ziebland [56], recommended values. Calculated values: ........... 
fifth-order classical calculation $[\eta]_{\mathrm{cl}, 5}$; potential by Aziz and Slaman [7]; - - - - - $\cdot$ potential by Cybulski and Toczylowski $[6] ;--$ -- potential by Wüest and Merkt [5]. 


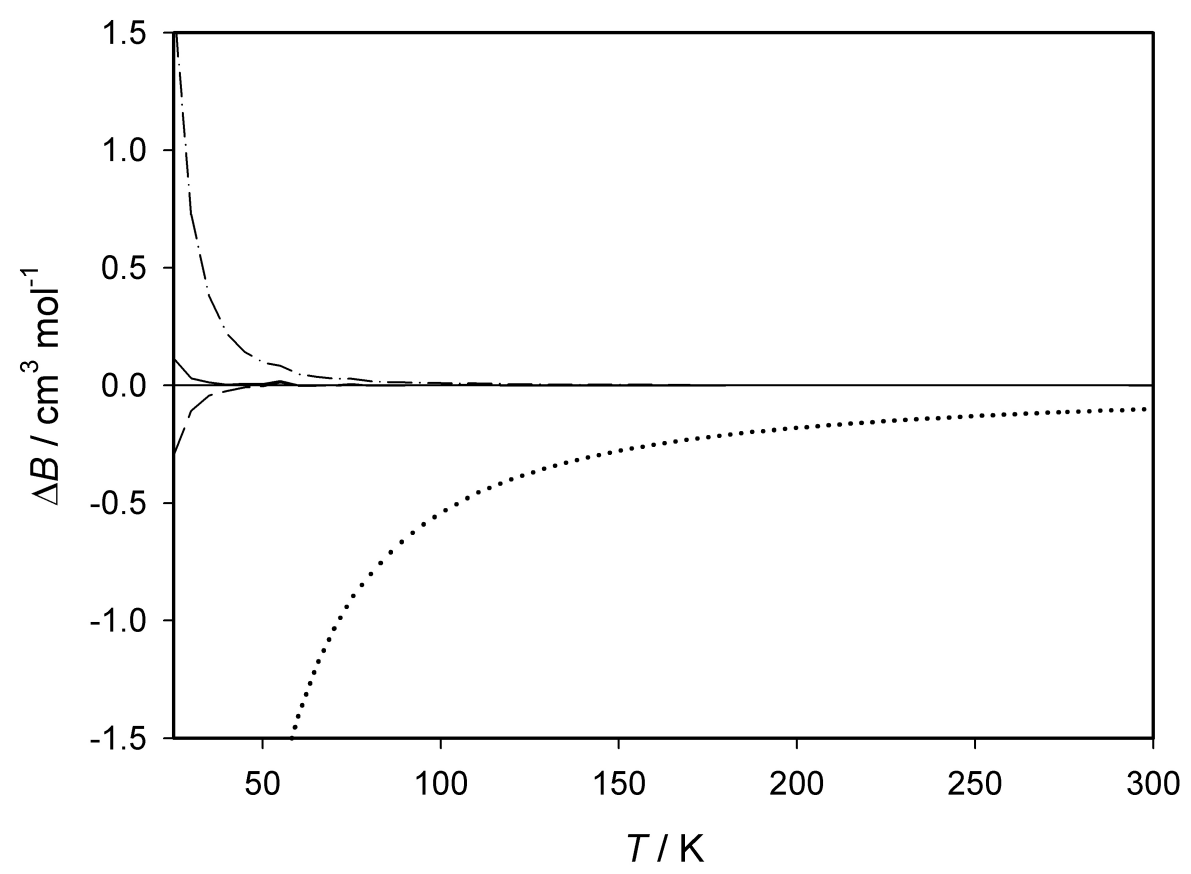

Figure 1. Differences $\Delta B=B_{\mathrm{qm}, \text { full }}-\left[B_{\mathrm{cl}}+\sum \lambda^{i} B_{\mathrm{qm}, i}\right]$ between the fully quantum-mechanically calculated values and the values resulting from the sum of a classical contribution and of different orders of quantum corrections to the second pressure virial coefficient for the new interatomic potential for Ne. Differences related to: $\cdots \cdots \cdots \cdot$. classical contribution $B_{\mathrm{cl}} ;-\cdot-\cdot-\cdot$ sum of classical contribution and of first-order quantum correction $B_{\mathrm{cl}}+\lambda B_{\mathrm{qm}, 1} ;----$ sum of classical contribution as well as of first-order and second-order quantum corrections $B_{\mathrm{cl}}+\lambda B_{\mathrm{qm}, 1}+\lambda^{2} B_{\mathrm{qm}, 2} ;-$ sum of classical contribution as well as of first-order, second-order and third-order quantum corrections

$$
B_{\mathrm{cl}}+\lambda B_{\mathrm{qm}, 1}+\lambda^{2} B_{\mathrm{qm}, 2}+\lambda^{3} B_{\mathrm{qm}, 3} .
$$




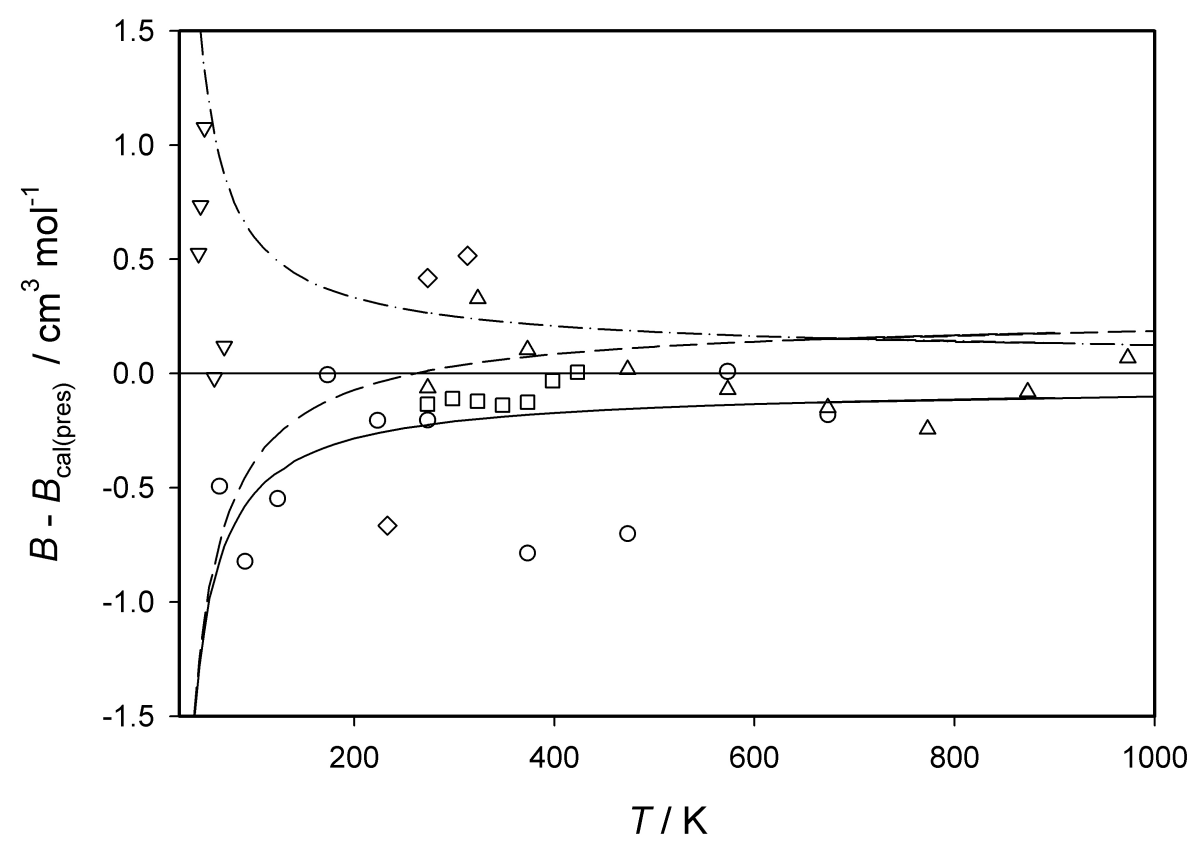

Figure 2. Differences $\left(B-B_{\text {cal }(\text { pres })}\right)$ of experimental $\left(B_{\exp }\right)$ and calculated $\left(B_{\text {cal }(\text { lit })}\right)$ second pressure virial coefficients from values $\left(B_{\text {cal }}\right.$ pres $)$ calculated with the new interatomic potential for Ne. Experimental data: $\bigcirc$ Holborn and Otto [19]; $\triangle$ Nicholson and Schneider [20]; $\square$ Michels et al. [21]; $\nabla$ Gibbons [22]; $\diamond \mathrm{Vogl}$ and Hall [23]. Calculated values: $\longrightarrow$ potential by Aziz and Slaman [7]; - . - . $-\cdot$ potential by Cybulski and Toczylowski [6]; - - - potential by Wüest and Merkt [5]. 
Figure 3. Comparison of experimental data and and of calculated values for the third pressure virial coefficient $C$ derived from the new interatomic potential for Ne. Experimental data: $\bigcirc$ Holborn and Otto [19]; $\triangle$ Nicholson and Schneider [20]; $\square$ Michels et al. [21]; $\nabla$ Gibbons [22]; $\diamond$ Vogl and Hall [23].

Calculated values: ---- classical contribution $C_{\text {add }},-\cdot-\cdot-\cdot$ classical and non-additivity contributions $C_{\text {add }}+C_{\text {non-add }}$, sum of classical and non-additivity contributions and of the first-order quantum correction $C_{\text {add }}+C_{\text {non-add }}+\lambda C_{\mathrm{qm}, 1}$. 
1

3

4

5

6

9

10

11

12

13

14

15

16

17

18

19

20

21

22

23

24

25

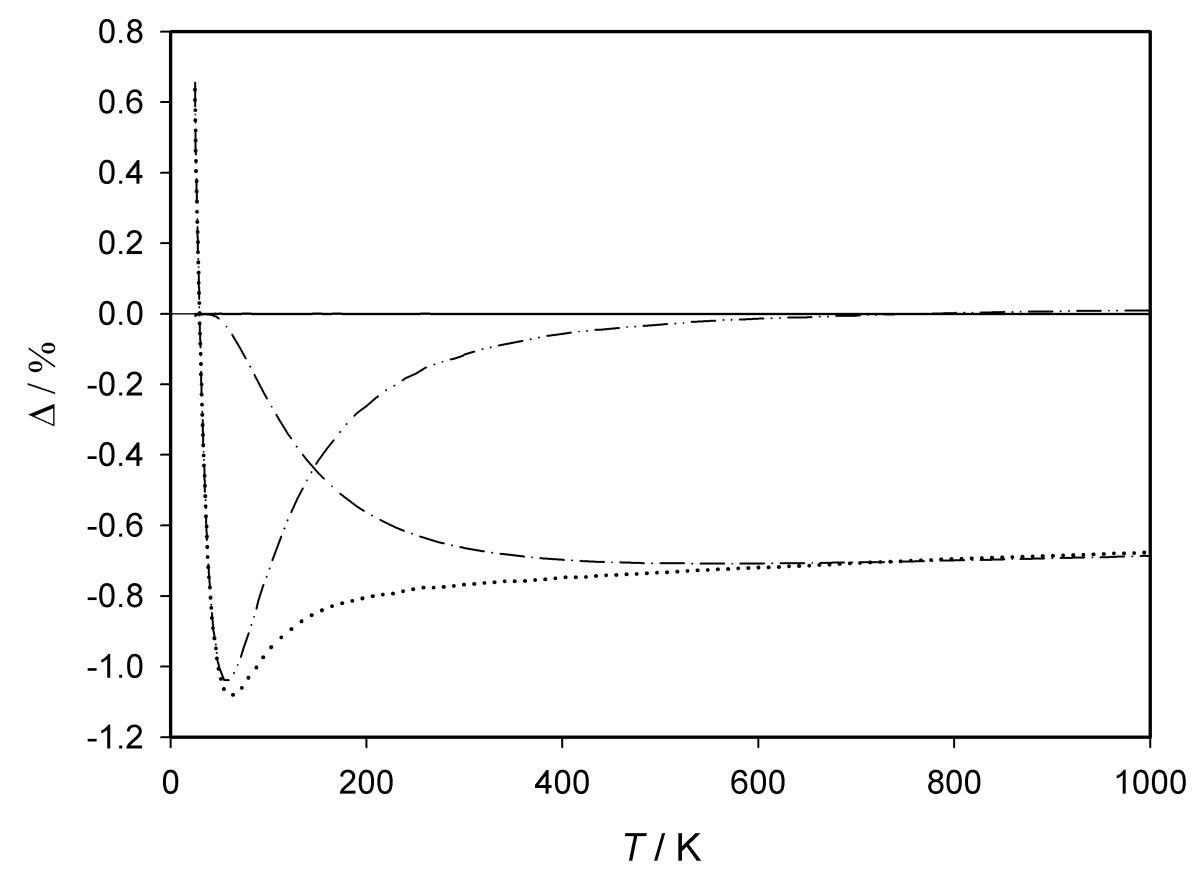

Figure 4. Relative deviations $\Delta=100\left(\eta-\eta_{\mathrm{qm}, 5}\right) / \eta_{\mathrm{qm}, 5}$ between viscosity values calculated for different approximation procedures and viscosity values resulting from quantum-mechanical calculations up to the fifth-order approximation for the individual $\left[\eta_{i j}\right]_{\mathrm{qm}, 5}$ within the first-order formulation of $\left[\eta_{\mathrm{mix}}\right]_{1}$ for the new interatomic potential for Ne. Differences related to: . . . . . . . first-order classical calculation $[\eta]_{\mathrm{cl}, 1} ;-\cdots-\cdots-\cdots$ fifth-order classical calculation $[\eta]_{\mathrm{cl}, 5} ;-\cdot-\cdots \cdot$ first-order quantum-mechanical calculation $\left[\eta_{\text {mix }}\right]_{\mathrm{qm}, 1}$. 


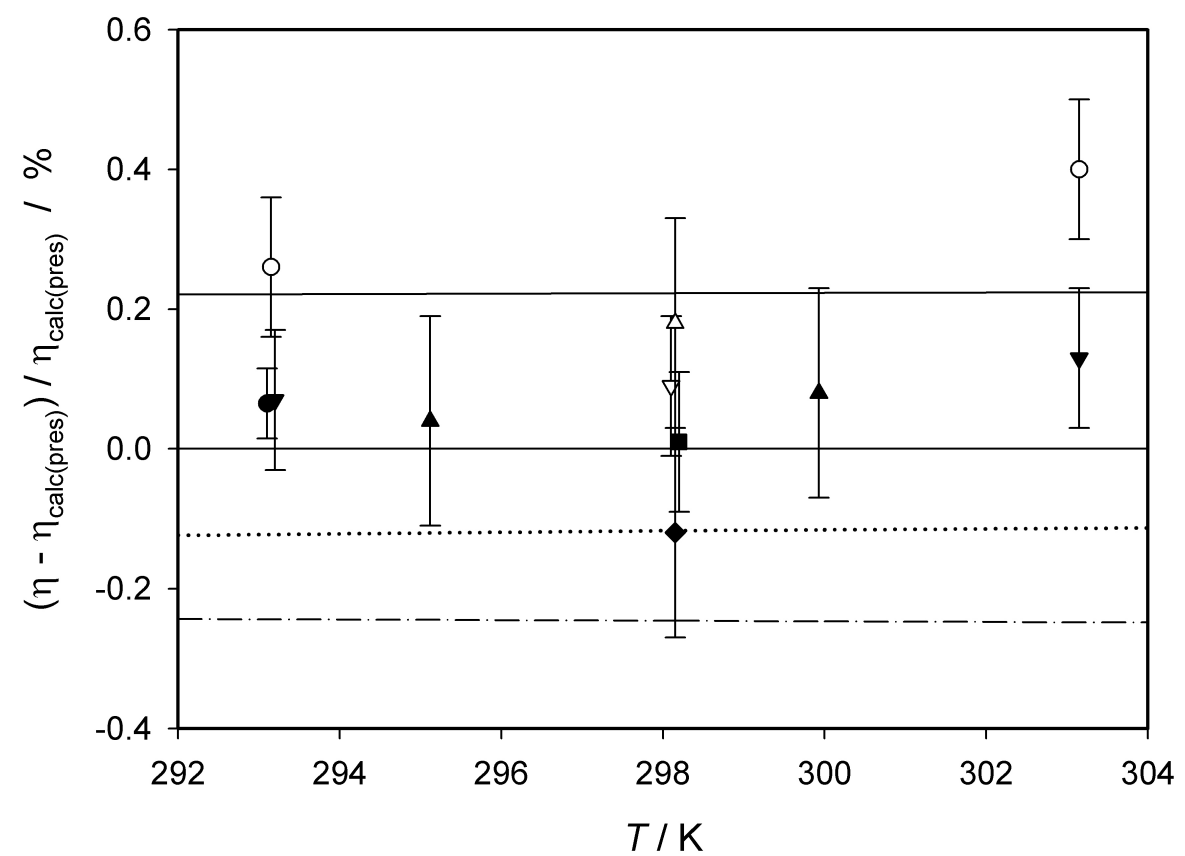

Figure 5. Relative deviations of experimental and calculated viscosity coefficients from values $\eta_{\text {cal(pres) }}$ calculated quantum-mechanically with the new interatomic potential for Ne near to room temperature. Experimental data with uncertainties characterized by error bars: $\bullet$ Kestin and Leidenfrost [24]; ○ Kestin and Nagashima [25]; $\mathbf{\nabla}$ DiPippo et al. [26]; $\nabla$ Kestin et al. [27], best estimate; $\boldsymbol{\square}$ Flynn et al. [28]; $\Delta$ Vogel [29], fitted value; $\boldsymbol{\Delta}$ Vogel [29], experimental data corrected according to new helium standard; Evers et al. [30]. Calculated values: $\cdots \cdots \cdots \cdots$ fifth-order classical calculation $[\eta]_{\mathrm{cl}, 5}$; potential by Aziz and Slaman [7];- - - - - p potential by Cybulski and Toczylowski [6].

2 3 
1

3

4

5

6

7

8

9

10

11

12

13

14

15

16

17

18

19

20

21

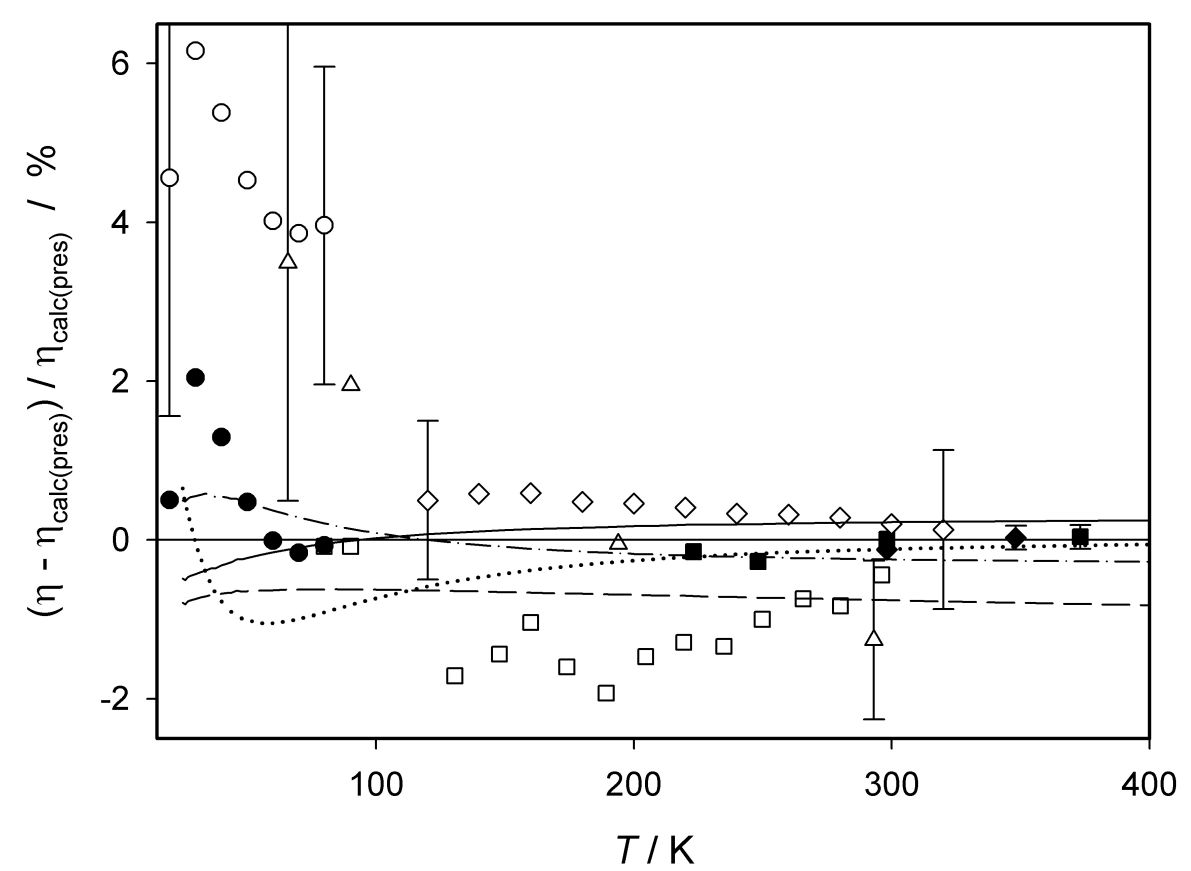

Figure 6. Relative deviations of experimental and calculated viscosity coefficients from values $\eta_{\text {cal(pres) }}$ calculated with the new interatomic potential for $\mathrm{Ne}$ at low and medium temperatures. Experimental data with uncertainties characterized by error bars: $\square$ Johnston and Grilly [32]; ○ Coremans et al. [33];

Coremans et al. [33], corrected according to new helium standard; $\triangle$ Rietveld et al. [34]; $\mathbf{\square}$ Flynn et

al. [28]; $\diamond$ Clarke and Smith [35]; $\diamond$ Evers et al. [30]. Calculated values: ........... fifth-order classical calculation $[\eta]_{\mathrm{cl}, 5} ; \longrightarrow$ potential by Aziz and Slaman $[7] ;-\cdot-\cdot-\cdot$ potential by Cybulski and Toczylowski [6]; - - - potential by Wüest and Merkt [5]. 


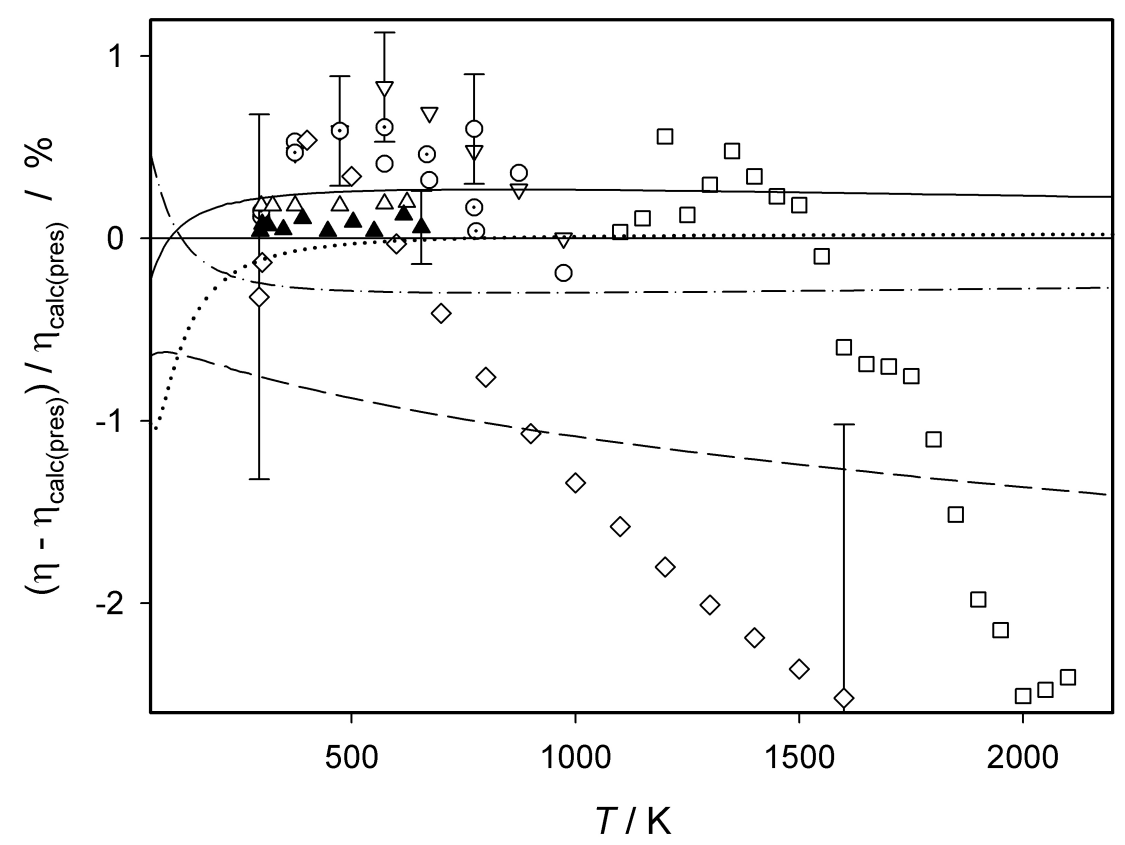

Figure 7. Deviations of experimental and calculated viscosity coefficients from values $\eta_{\text {cal(pres) }}$ calculated with the new interatomic potential for Ne at higher temperatures. Experimental data with uncertainties characterized by error bars: $\nabla$ Kestin et al. [27], best estimate; $\bigcirc$ Hellemans et al. [37]; $\odot$ Kestin et al. [38]; $\diamond$ Dawe and Smith [39]; $\square$ Guevara and Stensland [40]; $\triangle$ Vogel [29], fitted values; $\boldsymbol{\Delta}$ Vogel [29], experimental data corrected according to new helium standard. Calculated values: ... fifth-order classical calculation $[\eta]_{\mathrm{cl}, 5} ; \longrightarrow$ potential by Aziz and Slaman $[7] ;-\cdot-\cdots$ potential by Cybulski and Toczylowski [6]; - - - potential by Wüest and Merkt [5].

2

3

5

6

8

10

11
12

13

14

15

17

19

20 


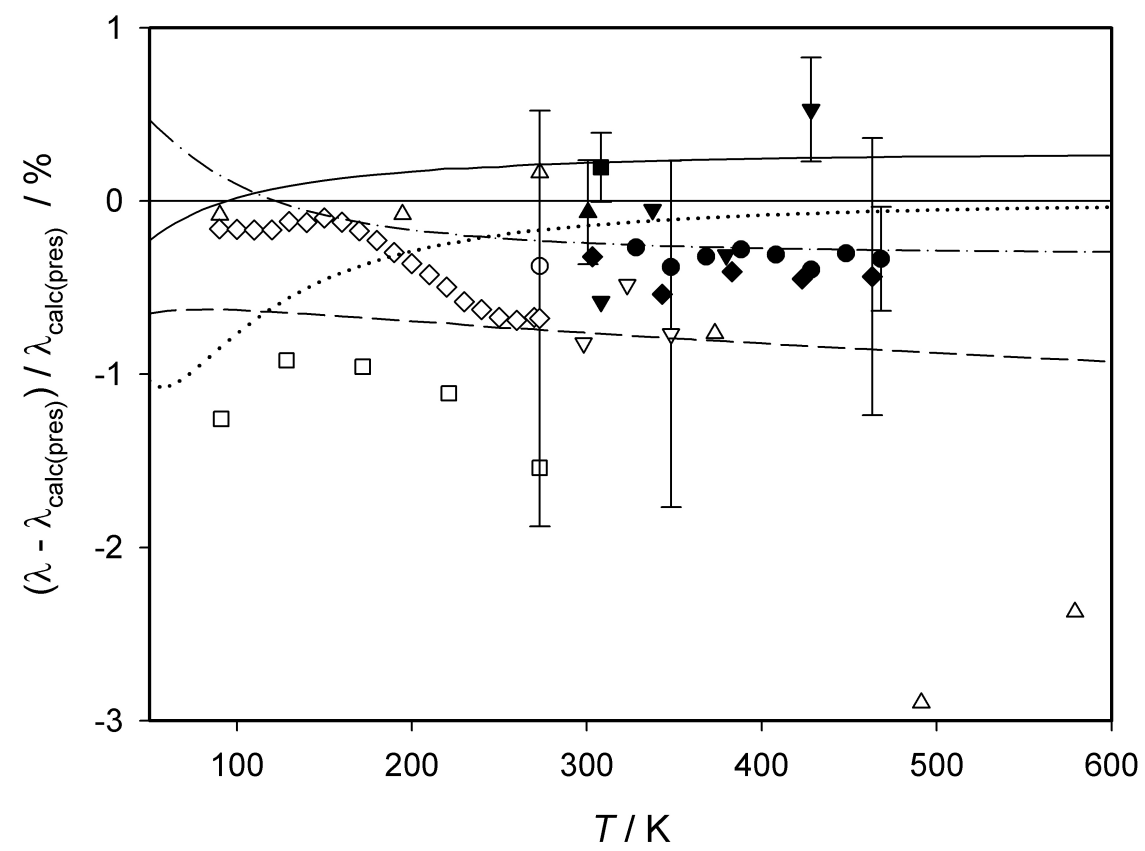

Figure 8. Deviations of experimental and calculated thermal conductivity coefficients from values $\lambda_{\text {cal(pres) }}$ calculated with the new interatomic potential for Ne at low and medium temperatures. Experimental data with uncertainties characterized by error bars: $\bullet$ Haarman [43]; $\boldsymbol{\Delta}$ Kestin et al. [44];

- Assael et al. [45]; $\boldsymbol{\nabla}$ Millat et al. [46]; Hemminger [47]; ○ Weber [48]; $\triangle$ Kannuluik and Carman [49]; $\square$ Keyes [50]; $\nabla$ Sengers et al. [51]; $\diamond$ Nesterov and Sudnik [52], smoothed values. Calculated values: ... fifth-order classical calculation $[\eta]_{\mathrm{cl}, 5} ;-$ potential by Aziz and Slaman $[7] ;-$ - - . . potential by Cybulski and Toczylowski [6]; - - - potential by Wüest and Merkt [5]. 


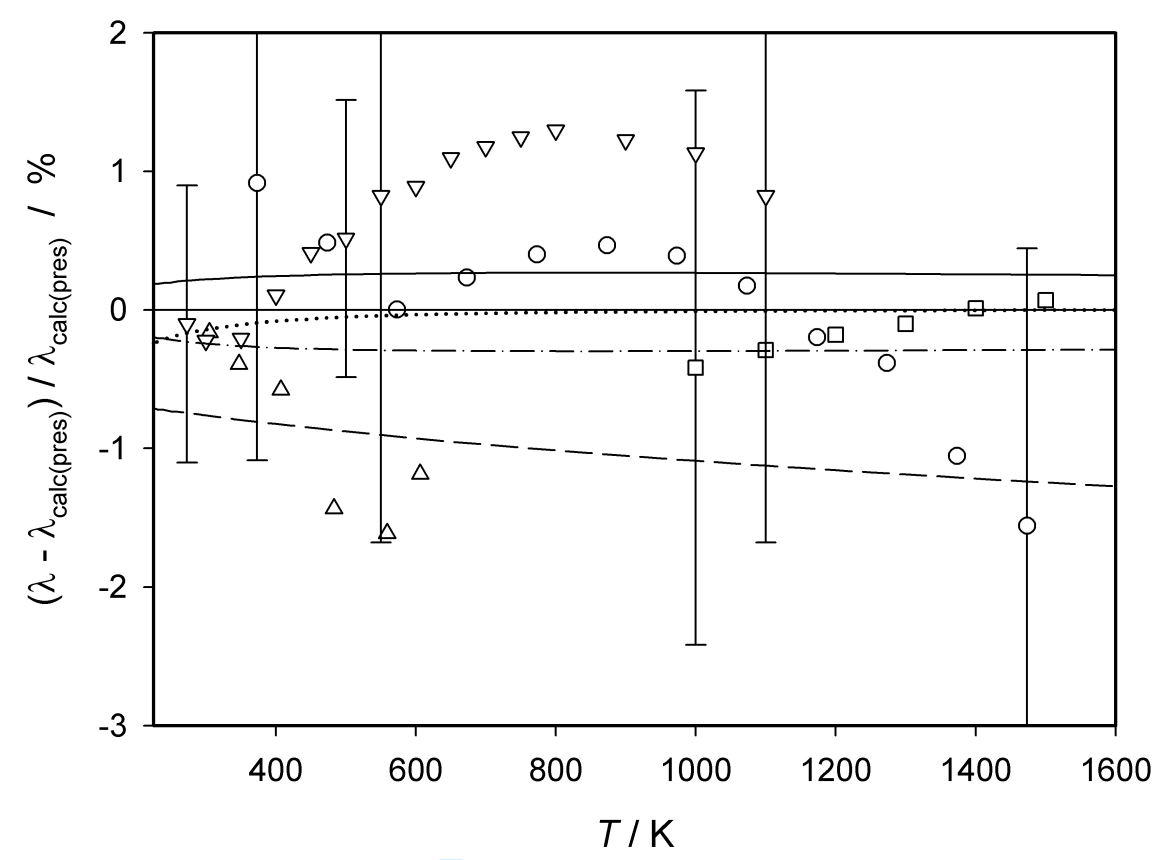

Figure 9. Deviations of experimental and calculated thermal conductivity coefficients from values $\lambda_{\text {cal(pres) }}$ calculated with the new interatomic potential for Ne at higher temperatures. Experimental data with uncertainties characterized by error bars: $\bigcirc$ Saxena and Saxena [53], smoothed values; $\triangle$ Tufeu et al. [54]; $\square$ Springer and Wingeier [55]; $\nabla$ Ziebland [56], recommended values. Calculated values: .. fifth-order classical calculation $[\eta]_{\mathrm{cl}, 5} ; \longrightarrow$ potential by Aziz and Slaman $[7] ;-$ - - . - . potential by Cybulski and Toczylowski [6]; - - - potential by Wüest and Merkt [5].

2

3

5

6

8

10

11

13

14

15
16

17

19

20

23

24

26 


\section{Page 33 of 44 \\ Molecular Physics}

\section{Appendix A: Thermophysical properties of neon calculated in this work}

The thermophysical properties of naturally occurring neon are given in table A.

Table A1. Thermophysical properties of neon for the interatomic potential of this work

\begin{tabular}{|c|c|c|c|c|}
\hline$T(\mathrm{~K})$ & $B\left(\mathrm{~cm}^{3} \mathrm{~mol}^{-1}\right)$ & $C\left(\mathrm{~cm}^{6} \mathrm{~mol}^{-2}\right)$ & $\eta(\mu \mathrm{Pas})$ & $\lambda\left(\mathrm{mW} \mathrm{m}^{-1} \mathrm{~K}^{-1}\right)$ \\
\hline 25.00 & -128.50 & -1716 & 3.9213 & 6.0597 \\
\hline 26.00 & -119.87 & -1130 & 4.0790 & 6.3033 \\
\hline 27.00 & -112.02 & -689.5 & 4.2353 & 6.5446 \\
\hline 28.00 & -105.04 & -358.4 & 4.3927 & 6.7878 \\
\hline 30.00 & -92.972 & 78.89 & 4.7097 & 7.2775 \\
\hline 32.00 & -82.953 & 325.4 & 5.0270 & 7.7676 \\
\hline 34.00 & -74.517 & 461.5 & 5.3447 & 8.2585 \\
\hline 36.00 & -67.316 & 532.2 & 5.6617 & 8.7482 \\
\hline 38.00 & -61.085 & 564.7 & 5.9784 & 9.2377 \\
\hline 40.00 & -55.657 & 574.0 & 6.2915 & 9.7214 \\
\hline 42.00 & -50.888 & 569.7 & 6.6035 & 10.204 \\
\hline 44.00 & -46.666 & 557.7 & 6.9132 & 10.682 \\
\hline 46.00 & -42.905 & 541.5 & 7.2191 & 11.155 \\
\hline 48.00 & -39.526 & 523.4 & 7.5245 & 11.627 \\
\hline 50.00 & -36.486 & 504.6 & 7.8257 & 12.093 \\
\hline 55.00 & -30.063 & 459.3 & 8.5658 & 13.237 \\
\hline 60.00 & -24.892 & 419.9 & 9.2862 & 14.352 \\
\hline 65.00 & -20.676 & 387.2 & 9.9868 & 15.436 \\
\hline 70.00 & -17.169 & 360.6 & 10.668 & 16.491 \\
\hline 75.00 & -14.213 & 339.9 & 11.331 & 17.518 \\
\hline 80.00 & -11.676 & 321.5 & 11.976 & 18.518 \\
\hline 85.00 & -9.4909 & 307.0 & 12.605 & 19.493 \\
\hline 90.00 & -7.5869 & 295.2 & 13.217 & 20.443 \\
\hline 95.00 & -5.9132 & 285.4 & 13.815 & 21.370 \\
\hline 100.00 & -4.4329 & 277.3 & 14.399 & 22.277 \\
\hline 110.00 & -1.9365 & 264.7 & 15.528 & 24.030 \\
\hline 120.00 & 0.0895 & 255.6 & 16.612 & 25.713 \\
\hline 130.00 & 1.7559 & 248.8 & 17.654 & 27.333 \\
\hline 140.00 & 3.1490 & 243.5 & 18.660 & 28.896 \\
\hline 150.00 & 4.3280 & 239.4 & 19.634 & 30.410 \\
\hline 160.00 & 5.3365 & 236.0 & 20.579 & 31.879 \\
\hline 170.00 & 6.2068 & 233.1 & 21.498 & 33.307 \\
\hline 180.00 & 6.9638 & 230.6 & 22.394 & 34.699 \\
\hline 190.00 & 7.6271 & 228.6 & 23.268 & 36.058 \\
\hline 200.00 & 8.2116 & 226.5 & 24.122 & 37.385 \\
\hline 210.00 & 8.7298 & 224.7 & 24.958 & 38.685 \\
\hline 220.00 & 9.1906 & 223.1 & 25.778 & 39.959 \\
\hline 230.00 & 9.6037 & 221.5 & 26.583 & 41.210 \\
\hline 240.00 & 9.9744 & 220.0 & 27.372 & 42.436 \\
\hline 250.00 & 10.308 & 218.6 & 28.149 & 43.643 \\
\hline 260.00 & 10.610 & 217.3 & 28.912 & 44.829 \\
\hline 270.00 & 10.884 & 215.9 & 29.665 & 45.999 \\
\hline 273.15 & 10.964 & 215.5 & 29.900 & 46.364 \\
\hline 280.00 & 11.133 & 214.6 & 30.408 & 47.153 \\
\hline 290.00 & 11.360 & 213.9 & 31.139 & 48.289 \\
\hline
\end{tabular}


34

Table A2. Table continued

\begin{tabular}{|c|c|c|c|c|}
\hline$T(\mathrm{~K})$ & $B\left(\mathrm{~cm}^{3} \mathrm{~mol}^{-1}\right)$ & $C\left(\mathrm{~cm}^{6} \mathrm{~mol}^{-2}\right)$ & $\eta(\mu \mathrm{Pa} \mathrm{s})$ & $\lambda\left(\mathrm{mW} \mathrm{m}^{-1} \mathrm{~K}^{-1}\right)$ \\
\hline 298.15 & 11.530 & 212.9 & 31.728 & 49.203 \\
\hline 300.00 & 11.567 & 212.2 & 31.860 & 49.410 \\
\hline 320.00 & 11.930 & 209.8 & 33.277 & 51.610 \\
\hline 340.00 & 12.237 & 207.4 & 34.660 & 53.758 \\
\hline 360.00 & 12.497 & 205.2 & 36.014 & 55.859 \\
\hline 380.00 & 12.719 & 203.0 & 37.339 & 57.917 \\
\hline 400.00 & 12.909 & 200.8 & 38.640 & 59.937 \\
\hline 420.00 & 13.072 & 198.8 & 39.918 & 61.921 \\
\hline 440.00 & 13.212 & 196.7 & 41.174 & 63.871 \\
\hline 460.00 & 13.333 & 194.8 & 42.411 & 65.789 \\
\hline 480.00 & 13.437 & 192.8 & 43.628 & 67.679 \\
\hline 500.00 & 13.527 & 190.9 & 44.829 & 69.542 \\
\hline 550.00 & 13.700 & 186.4 & 47.761 & 74.091 \\
\hline 600.00 & 13.819 & 182.1 & 50.604 & 78.502 \\
\hline 650.00 & 13.896 & 178.0 & 53.370 & 82.793 \\
\hline 700.00 & 13.943 & 174.2 & 56.068 & 86.977 \\
\hline 750.00 & 13.968 & 170.6 & 58.703 & 91.065 \\
\hline 800.00 & 13.975 & 167.2 & 61.284 & 95.067 \\
\hline 850.00 & 13.968 & 163.9 & 63.814 & 98.9901 \\
\hline 900.00 & 13.952 & 160.8 & 66.298 & 102.84 \\
\hline 950.00 & 13.927 & 157.9 & 68.739 & 106.63 \\
\hline 1000.00 & 13.895 & 155.1 & 71.141 & 110.35 \\
\hline 1100.00 & 13.819 & 149.9 & 75.838 & 117.63 \\
\hline 1200.00 & 13.730 & 145.1 & 80.408 & 124.72 \\
\hline 1300.00 & 13.634 & 140.7 & 84.866 & 131.63 \\
\hline 1400.00 & 13.534 & 136.7 & 89.223 & 138.38 \\
\hline 1500.00 & 13.432 & 132.9 & 93.489 & 144.99 \\
\hline 1600.00 & 13.329 & 129.5 & 97.674 & 151.48 \\
\hline 1700.00 & 13.226 & 126.2 & 101.78 & 157.85 \\
\hline 1800.00 & 13.125 & 123.2 & 105.83 & 164.11 \\
\hline 1900.00 & 13.025 & 120.3 & 109.80 & 170.28 \\
\hline 2000.00 & 12.926 & 117.6 & 113.72 & 176.35 \\
\hline 2100.00 & 12.830 & 115.1 & 117.59 & 182.34 \\
\hline 2200.00 & 12.736 & 112.7 & 121.40 & 188.25 \\
\hline 2300.00 & 12.644 & 110.4 & 125.17 & 194.08 \\
\hline 2400.00 & 12.554 & 108.2 & 128.89 & 199.85 \\
\hline 2500.00 & 12.467 & 106.2 & 132.57 & 205.55 \\
\hline 2600.00 & 12.381 & 104.2 & 136.21 & 211.19 \\
\hline 2700.00 & 12.298 & 102.4 & 139.81 & 216.76 \\
\hline 2800.00 & 12.216 & 100.6 & 143.38 & 222.29 \\
\hline 2900.00 & 12.137 & 98.92 & 146.91 & 227.76 \\
\hline 3000.00 & 12.059 & 97.29 & 150.41 & 233.18 \\
\hline 3100.00 & 11.984 & 95.73 & 153.87 & 238.55 \\
\hline 3200.00 & 11.910 & 94.24 & 157.31 & 243.88 \\
\hline 3300.00 & 11.838 & 92.80 & 160.72 & 249.16 \\
\hline 3400.00 & 11.767 & 91.41 & 164.11 & 254.40 \\
\hline 3500.00 & 11.698 & 90.08 & 167.46 & 259.60 \\
\hline 3600.00 & 11.631 & 88.80 & 170.79 & 264.76 \\
\hline 3700.00 & 11.565 & 87.56 & 174.10 & 269.88 \\
\hline 3800.00 & 11.501 & 86.36 & 177.39 & 274.97 \\
\hline 3900.00 & 11.438 & 85.21 & 180.65 & 280.02 \\
\hline 4000.00 & 11.376 & 84.09 & 183.89 & 285.04 \\
\hline
\end{tabular}


Table A3. Table continued

\begin{tabular}{rcccc}
\hline$T(\mathrm{~K})$ & $B\left(\mathrm{~cm}^{3} \mathrm{~mol}^{-1}\right)$ & $C\left(\mathrm{~cm}^{6} \mathrm{~mol}^{-2}\right)$ & $\eta(\mu \mathrm{Pas})$ & $\lambda\left(\mathrm{mW} \mathrm{m}^{-1} \mathrm{~K}^{-1}\right)$ \\
\hline 4100.00 & 11.316 & 83.01 & 187.11 & 290.03 \\
4200.00 & 11.257 & 81.96 & 190.31 & 294.99 \\
4300.00 & 11.199 & 80.95 & 193.49 & 299.91 \\
4400.00 & 11.142 & 79.96 & 196.65 & 304.81 \\
4500.00 & 11.087 & 79.01 & 199.80 & 309.68 \\
4600.00 & 11.032 & 78.09 & 202.92 & 314.52 \\
4700.00 & 10.979 & 77.19 & 206.03 & 319.33 \\
4800.00 & 10.926 & 76.31 & 209.12 & 324.12 \\
4900.00 & 10.875 & 75.46 & 212.20 & 328.88 \\
5000.00 & 10.825 & 74.64 & 215.26 & 333.62 \\
6000.00 & 10.366 & 67.45 & 245.09 & 379.81 \\
7000.00 & 9.9770 & 61.77 & 273.72 & 424.15 \\
8000.00 & 9.6396 & 57.14 & 301.40 & 467.00 \\
9000.00 & 9.3429 & 53.30 & 328.28 & 508.61 \\
10000.00 & 9.0788 & 50.05 & 354.48 & 549.16 \\
\hline
\end{tabular}




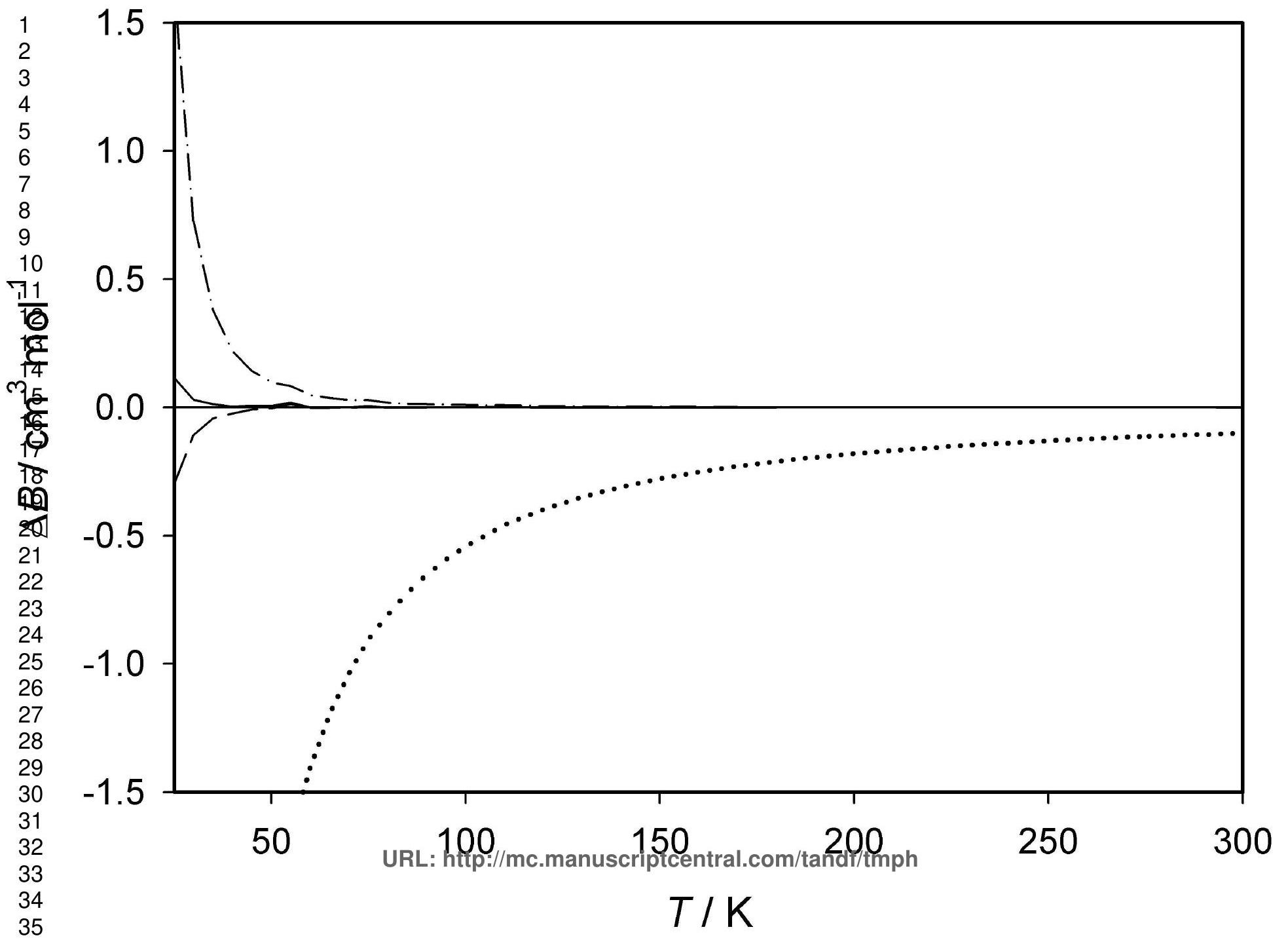




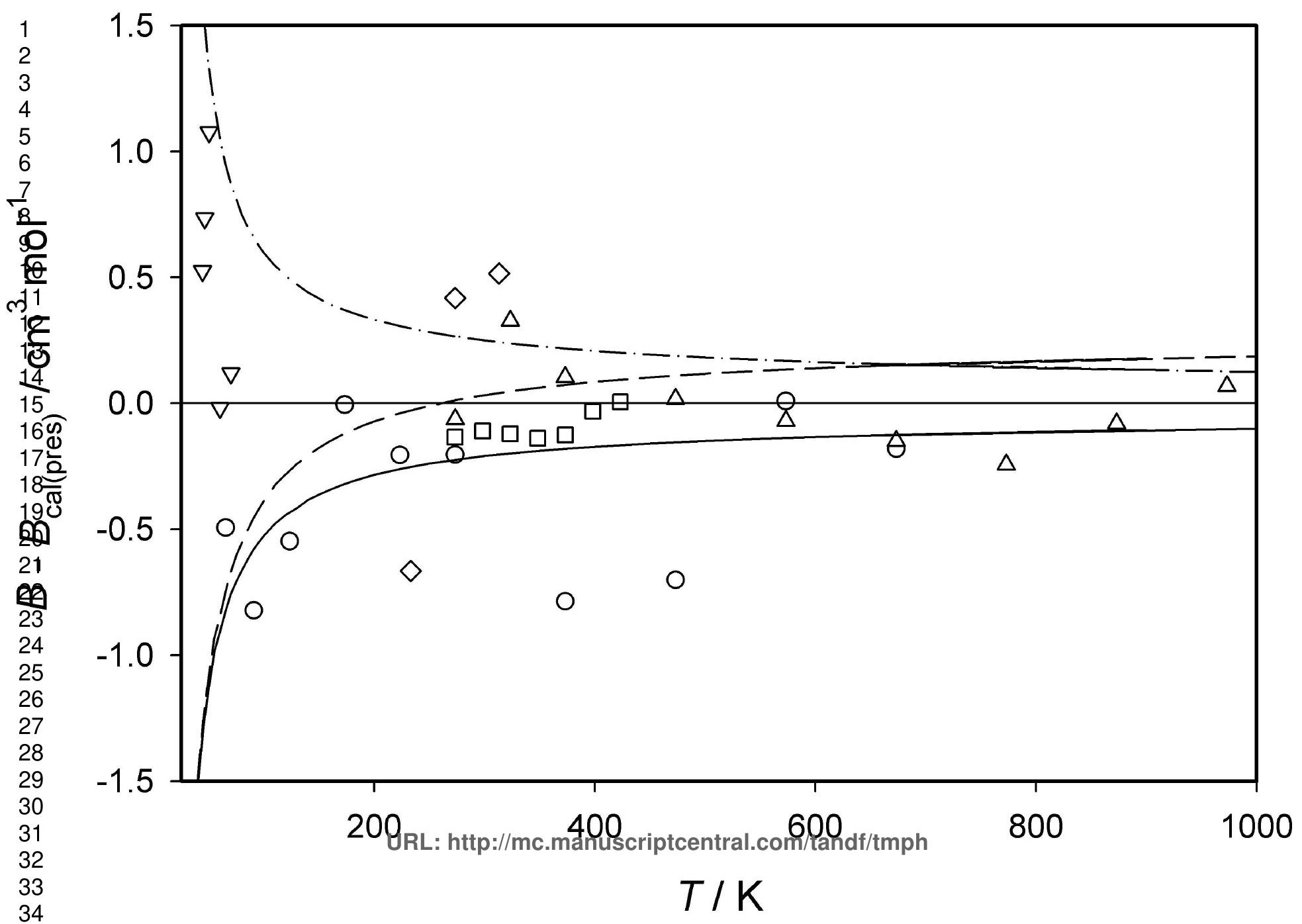




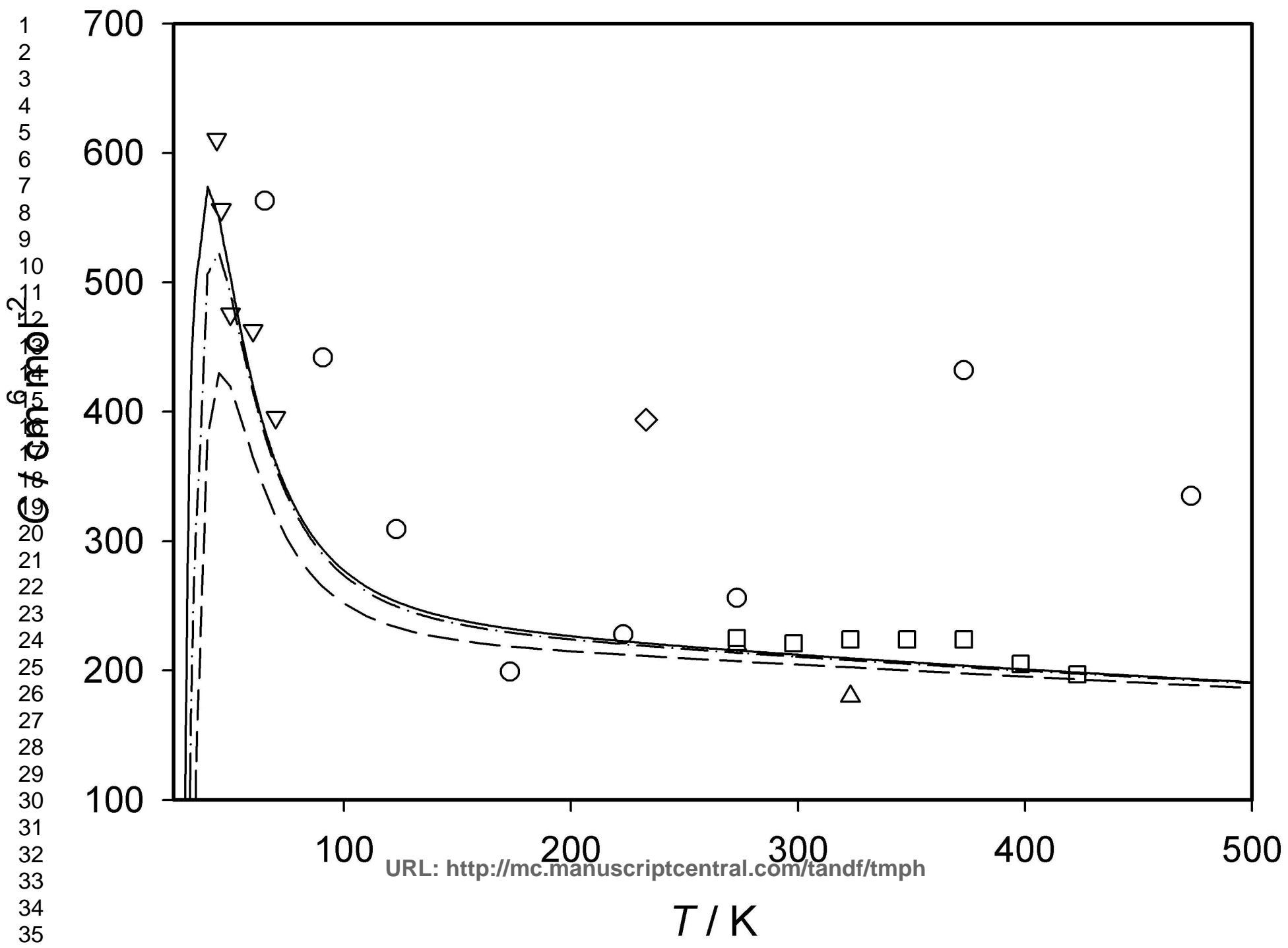




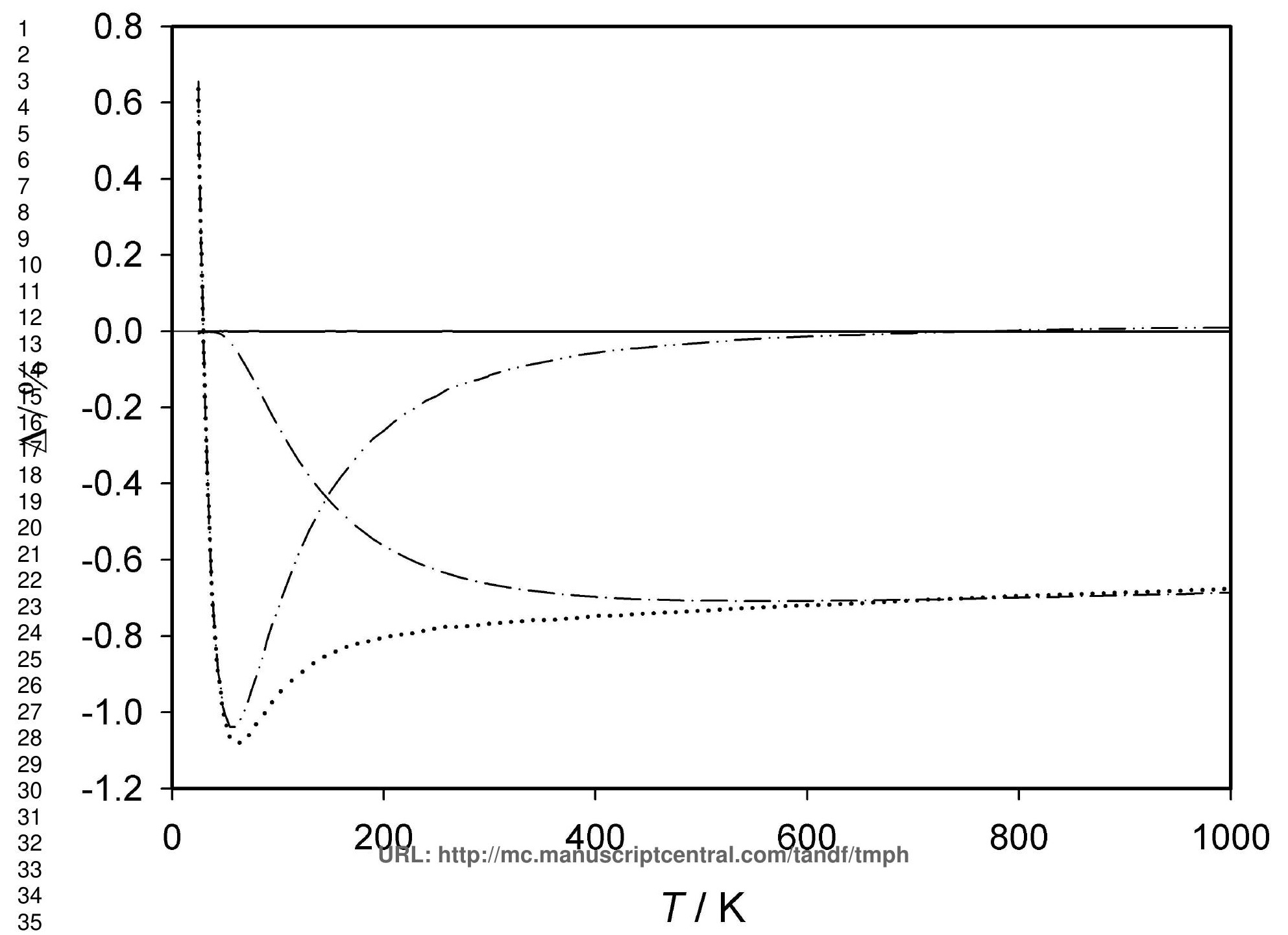




\section{Page 43 of 44}

Molecular Physics

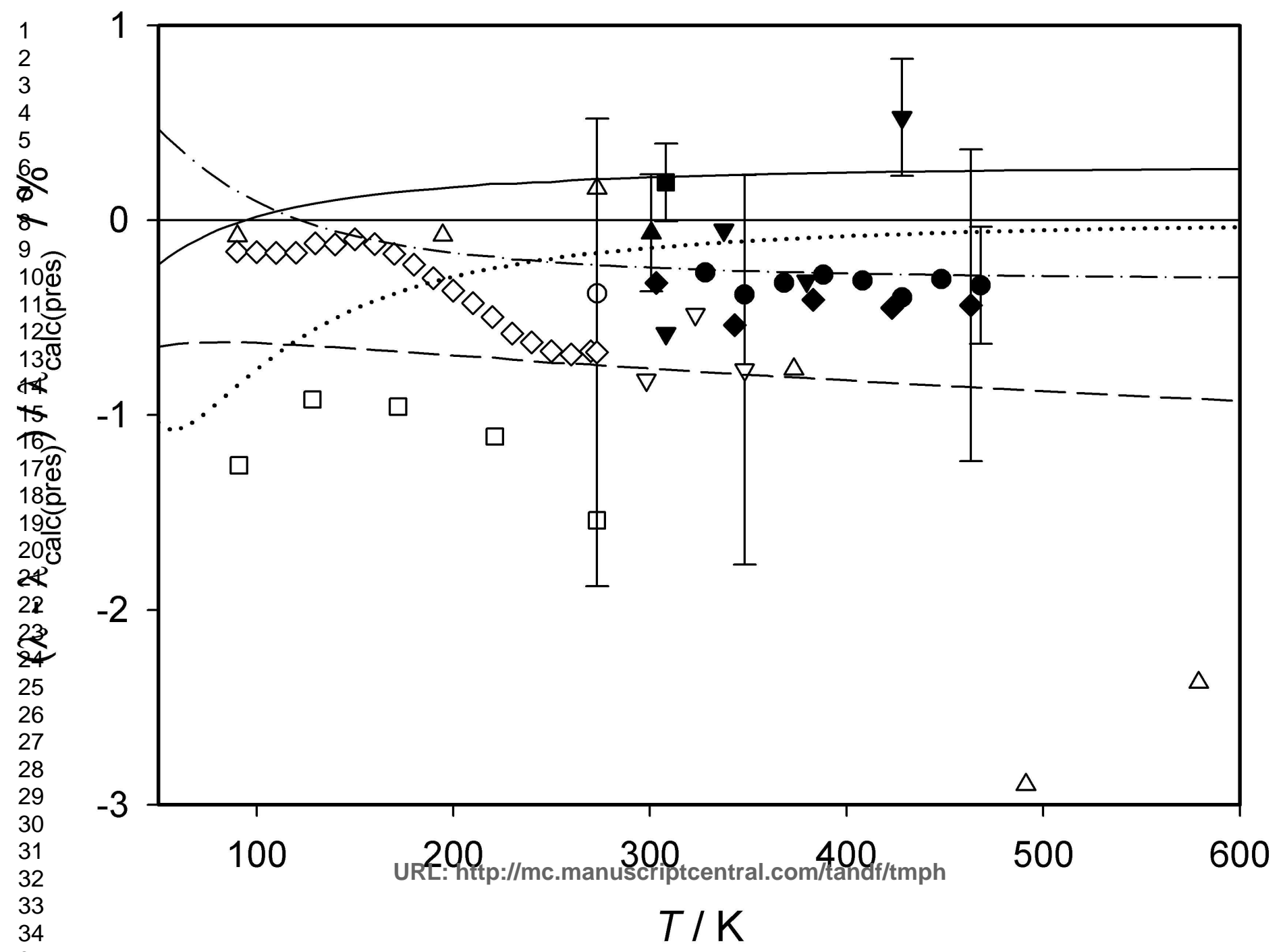


